\title{
THE SELBERG TRACE FORMULA. VIII, CONTRIBUTION FROM THE CONTINUOUS SPECTRUM
}

\author{
M. SCOTT OSBORNE AND GARTH WARNER
}

\begin{abstract}
The purpose of this paper is to isolate the contribution from the
\end{abstract} continuous spectrum to the Selberg trace formula.

\section{INTRODUCTION}

This is the eighth in a projected series of papers in which we plan to come to grips with the Selberg trace formula, the ultimate objective being a reasonably explicit expression. In a word, our purpose here is to complete that aspect of the investigation focusing on the contribution to the trace arising from the continuous spectrum, call it

$$
\operatorname{Con}-\operatorname{Sp}(\alpha: \Gamma) \text {. }
$$

We shall see that $\operatorname{Con}-\operatorname{Sp}(\alpha: \Gamma)$ is equal to

$$
\sum_{\mathscr{C}_{i_{0}}} \sum_{\mathscr{O}_{i_{0}}} \sum_{w_{i_{0}}} \operatorname{Con}-\mathbf{S p}\left(\alpha: \Gamma: \mathscr{C}_{i_{0}}, \mathscr{O}_{i_{0}}, w_{i_{0}}\right),
$$

where the ingredients of the triple $\left(\mathscr{C}_{i_{0}}, \mathscr{O}_{i_{0}}, w_{i_{0}}\right)$ are

$$
\left\{\begin{array}{l}
\text { a proper } G \text {-conjugacy class } \mathscr{C}_{i_{0}} \text { of } \Gamma \text {-cuspidals, } \\
\text { an orbit type } \mathscr{O}_{i_{0}}, \\
\text { a simple } w_{i_{0}} \text { of the ambient Weyl group. }
\end{array}\right.
$$

The main result of this paper is a formula for

$$
\operatorname{Con-Sp}\left(\alpha: \Gamma: \mathscr{C}_{i_{0}}, \mathscr{O}_{i_{0}}, w_{i_{0}}\right)
$$

involving an integral over $\sqrt{-1} \operatorname{Ker}\left(1-w_{i_{0}}\right)$ of an object formed from familiar ingredients: c-functions, exponentials, and Ind-functions.

[Note: Strictly speaking, there is an additional ingredient present in the formula, namely a parameter $\mathbf{H}$ from the truncation space that, however, we shall ignore for the purposes of this Introduction.]

Received by the editors March 17, 1989.

1980 Mathematics Subject Classification (1985 Revision). Primary 11 F72.

The authors' research was supported in part by the National Science Foundation. 
Our approach to this conclusion is essentially combinatorial. So, we start off in $\S 2$ with a result that tells us how $A$-transforms behave when applied to a product, thus augmenting and supplementing the machinery from $\S \S 3-4$ of [11]. In $\S 3$, we recall an important theorem due to Arthur [4]. Roughly speaking, it asserts that for the analysis one can throw away the special $W$ that do not contain $\operatorname{Ker}\left(1-w_{i_{0}}\right)$. The subject of $\S 4$ is the cancellation, culminating in Theorem 4.3. Using this, in $\S 5$ we isolate $\operatorname{Con}-\operatorname{Sp}(\alpha: \Gamma)$. Finally, we finish up in $\S 6$ by proving that the term initially ignored is in fact ignorable as it disappears when the truncation parameter is sent to infinity.

An earlier version of this paper was circulated a while back in preprint form. In it, we looked at the same problem in a very different way, namely by means of the Dini calculus. To get a handle on $\operatorname{Con}-\operatorname{Sp}(\alpha: \Gamma)$, we had to formulate a conjecture that, unfortunately, we were unable to establish in general. The present effort is therefore a substantial improvement over its forerunner in that, apart from the usual assumptions and conventions (cf. [11, §9]), its conclusions are "conjecture free" and totally explicit. In addition, as a bonus the conjecture from the earlier version turns out to be a corollary of what is said here, a point of some interest even though the Dini calculus no longer seems to be the way to go.

As a general reference and suggested overall introduction to the subject, we shall use our monograph The Theory of Eisenstein Systems, Academic Press, New York, 1981. Throughout the sequel, the title of this work has been abbreviated to TES.

\section{The $A$-TRANSFORM OF A PRODUCT}

The purpose of this section is to determine the behavior of the $A$-transform $[8, \S 2]$ under the formation of products. As a byproduct of this study, we shall recover a result of Arthur $[2, \S 6]$ that will be employed later on.

As always, we shall work within the context of a geometric g.r.s. $(V, \Phi)$, using without comment the notation and theory set down in [8].

This said, attached to a given $\phi$ is its $A$-transform $A_{\phi}$. To proceed, a relative version of this notion will be required. Thus, suppose that $F_{1} \subset F_{2}$. Then

$$
V=V\left(F_{1}\right) \oplus V_{F_{2}} \oplus V_{F_{1}} \cap V\left(F_{2}\right),
$$

so a given $\Lambda \in \sqrt{-1} V$ decomposes as

$$
\Lambda=\Lambda\left(F_{1}\right)+\Lambda_{F_{2}}+\Lambda_{F_{1}}\left(F_{2}\right) .
$$

Generically, denote by $A_{F_{1}}\left(F_{2}\right)$ the $A$-transform per $V_{F_{1}} \cap V\left(F_{2}\right)$. Writing

$$
\phi(\Lambda)=\phi\left(\Lambda\left(F_{1}\right)+\Lambda_{F_{2}}: \Lambda_{F_{1}}\left(F_{2}\right)\right),
$$

put

$$
A_{\phi}^{F_{1}, F_{2}}(\Lambda)=\left[A_{F_{1}}\left(F_{2}\right)\right]_{\phi\left(\Lambda_{F_{2}}: ?\right)}\left(\Lambda_{F_{1}}\left(F_{2}\right)\right) .
$$

Observe that $\Lambda\left(F_{1}\right)$ has been forgotten: $A_{\phi}^{F_{1}, F_{2}}(\Lambda)$ is a function of $\Lambda_{F_{1}}$ alone. The main result is then: 
Theorem 2.1. Given $\phi$ and $\psi$,

$$
A_{\phi \psi}=\sum_{F} A_{\phi}^{*, F} \bullet A_{\psi}^{F, *} .
$$

To prove this, we can assume without loss of generality that $\phi, \psi \in$ $\mathscr{C}(\sqrt{-1} V)$, say

$$
\phi=\hat{f}, \quad f \in \mathscr{C}(V), \quad \psi=\hat{g}, \quad g \in \mathscr{C}(V) .
$$

On general grounds (cf. $[8, \S 2])$,

$$
A_{\phi \psi}=A_{\hat{f} \hat{g}}=A_{\widehat{f * g}}=(-1)^{l} \widehat{\Gamma}_{f * g},
$$

$\Gamma_{f * g}$ is the $\Gamma$-transform of $f * g$, i.e.,

$$
\Gamma_{f * g}(H)=\int_{V} \Gamma_{*}^{*}\left(H, H_{0}\right) f * g\left(H_{0}\right) d H_{0} .
$$

Now

$$
\begin{aligned}
\int_{V} \Gamma_{*}^{*}\left(H, H_{0}\right) f * g\left(H_{0}\right) d H_{0} \\
\quad=\int_{V} \int_{V} \Gamma\left(H, H_{1}\right) f\left(H_{1}-H_{2}\right) g\left(H_{2}\right) d H_{1} d H_{2} \\
=\int_{V} \int_{V} \Gamma\left(H, H_{1}+H_{2}\right) f\left(H_{1}\right) g\left(H_{2}\right) d H_{1} d H_{2} \\
=\sum_{F} \int_{V} \int_{V} \Gamma_{*}^{F}\left(H, H_{1}\right) \Gamma_{F}^{*}\left(H-H_{1}, H_{2}\right) f\left(H_{1}\right) g\left(H_{2}\right) d H_{1} d H_{2},
\end{aligned}
$$

where, in passing to the last equality, we have invoked Proposition 3.1 of [11]. Accordingly,

$$
\begin{aligned}
& A_{\phi \psi}(\Lambda)=(-1)^{l} \sum_{F} \int_{V} \int_{V} \int_{V} e^{(\Lambda, H)} \Gamma_{*}^{F}\left(H, H_{1}\right) \Gamma_{F}^{*}\left(H-H_{1}, H_{2}\right) \\
& \times f\left(H_{1}\right) g\left(H_{2}\right) d H d H_{1} d H_{2} \\
&=(-1)^{l} \sum_{F} \int_{V} \int_{V} \int_{V} e^{\left(\Lambda, H+H_{1 F}\right)} \Gamma_{*}^{F}\left(H, H_{1}\right) \Gamma_{F}^{*}\left(H, H_{2}\right) \\
&=(-1)^{l} \sum_{F} \int_{V(F)} \int_{V_{F}} \int_{V} \int_{V} e^{\left(\Lambda, H(F)+H_{F}+H_{1 F}\right)} \Gamma_{*}^{F}\left(H(F), H_{1}\right) \Gamma_{F}^{*}\left(H_{F}, H_{2}\right) \\
& \times f\left(H_{1}\right) g\left(H_{2}\right) d H(F) d H_{F} d H_{1} d H_{2} \\
&=(-1)^{l} \sum_{F} I(F) \bullet I I_{F}
\end{aligned}
$$

if

$$
I(F)=\int_{V(F)} \int_{V} e^{\left(\Lambda, H(F)+H_{1 F}\right)} \Gamma_{*}^{F}\left(H(F), H_{1}\right) f\left(H_{1}\right) d H_{1} d H(F)
$$


and

$$
I I_{F}=\int_{V_{F}} \int_{V} e^{\left(\Lambda, H_{F}\right)} \Gamma_{F}^{*}\left(H_{F}, H_{2}\right) g\left(H_{2}\right) d H_{2} d H_{F} .
$$

Fix an $F$ and consider separately $I(F)$ and $I I_{F}$. From the definitions, on the one hand,

$$
\begin{aligned}
& \int_{V(F)} \int_{V} e^{\left(\Lambda, H(F)+H_{1 F}\right)} \Gamma_{*}^{F}\left(H(F), H_{1}\right) f\left(H_{1}\right) d H_{1} d H(F) \\
& =\int_{V(F)} \int_{V(F)} e^{(\Lambda(F), H(F))} \Gamma_{*}^{F}\left(H(F), H_{1}(F)\right) \\
& \quad \quad \quad \quad \int_{V_{F}} e^{\left(\Lambda_{F}, H_{1 F}\right)} f\left(H_{1}(F)+H_{1 F}\right) d H_{1 F} d H_{1}(F) d H(F) \\
& =(-1)^{\#(F)}\left[A_{*}(F)\right]_{\phi\left(\Lambda_{F}: ?\right)}(\Lambda(F)) \\
& =(-1)^{\#(F)} A_{\phi}^{*, F}(\Lambda)
\end{aligned}
$$

while, on the other hand,

$$
\begin{aligned}
& \int_{V_{F}} \int_{V} e^{\left(\Lambda, H_{F}\right)} \Gamma_{F}^{*}\left(H_{F}, H_{2}\right) g\left(H_{2}\right) d H_{2} d H_{F} \\
& =\int_{V_{F}} \int_{V_{F}} e^{\left(\Lambda_{F}, H_{F}\right)} \Gamma_{F}^{*}\left(H_{F}, H_{2 F}\right) \\
& \quad \quad \times \int_{V(F)} g\left(H_{2}(F)+H_{2 F}\right) d H_{2}(F) d H_{2 F} d H_{F} \\
& =(-1)^{l-\#(F)}\left[A_{F}(*)\right]_{\psi(0: ?)}\left(\Lambda_{F}\right) \\
& =(-1)^{l-\#(F)} A_{\psi}^{F, *}(\Lambda) .
\end{aligned}
$$

Therefore

$$
\begin{aligned}
A_{\phi \psi}(\Lambda) & =(-1)^{l} \sum_{F} I(F) \bullet I I_{F} \\
& =(-1)^{l} \sum_{F}\left((-1)^{\#(F)} A_{\phi}^{*, F}(\Lambda)\right) \bullet\left((-1)^{l-\#(F)} A_{\psi}^{F, *}(\Lambda)\right) \\
& =\sum_{F} A_{\phi}^{*, F}(\Lambda) \bullet A_{\psi}^{F, *}(\Lambda),
\end{aligned}
$$

as desired.

Remark. The evaluation of $I(F)$ and $I I_{F}$ supra is embodied in the rule

$$
A_{\hat{h}}^{F_{1}, F_{2}}(\Lambda)=(-1)^{\#\left(F_{2}-F_{1}\right)} \int_{V_{F_{1}} \cap V\left(F_{2}\right)} \int_{V} e^{\left(\Lambda, H^{\prime}+H_{F_{2}}\right)} \Gamma_{F_{1}}^{F_{2}}\left(H^{\prime}, H\right) h(H) d H d H^{\prime}
$$

the verification of which is immediate.

Suppose that $\phi=\left\{\phi_{\mathscr{C}}\right\}$ is a $C^{\infty}$ Detroit family. Then we may form $\amalg_{\phi}$ and $A_{\phi}$, functions that can be shown to be the same (cf. Theorem 4.4 of [8]). 
Now let $\phi$ and $\psi$ be two $C^{\infty}$ Detroit families. Then the product $\phi \bullet \psi$ is a $C^{\infty}$ Detroit family and, utilizing the formalism of [11, $\left.\S 4\right]$,

$$
\begin{aligned}
\amalg_{\phi \bullet \psi}(\Lambda) & =A_{\phi \bullet \psi}(\Lambda)=\sum_{\mathscr{E}} A_{\phi_{\mathscr{E}} \bullet \psi_{\mathscr{E}}}(\Lambda) \\
& =\sum_{\mathscr{E}} \sum_{\left\{F: F \subset F_{0}(\mathscr{C})\right\}} A_{\phi_{\mathscr{E}}}^{*, F}(\Lambda) \bullet A_{\psi_{\mathscr{E}}}^{F, *}(\Lambda) \\
& =\sum_{W} \sum_{\mathscr{E}_{W}} \sum_{\mathscr{E}(W)} A_{\phi_{\mathscr{E}}}^{*, F_{0}(\mathscr{E}(W))}(\Lambda) \bullet A_{\psi_{\mathscr{Z}}}^{F_{0}(\mathscr{E}(W)), *}\left(\Lambda_{W}\right) .
\end{aligned}
$$

Thanks to Lemma 4.1 of [8], $A_{\psi_{\mathscr{E}}}^{F_{0}(\mathscr{E}(W)), *}\left(\Lambda_{W}\right)$ is independent of $\mathscr{C}(W)$, call it $A_{\psi, \mathscr{B}_{W}}(\Lambda)$. To interpret the sum over $\mathscr{C}(W)$ of what is left, observe that Lemma 4.2 of [8] implies that the prescription

$$
\phi_{\mathscr{E}(W)}(\Lambda(W))=\phi_{\mathscr{E}}\left(\Lambda(W)+\Lambda_{W}\right)
$$

determines a Detroit family $\phi\left(\mathscr{C}_{W}\right)$ on $W$, so

$$
\sum_{\mathscr{B}(W)} A_{\phi_{\mathscr{C}}}^{*, F_{0}(\mathscr{E}(W))}(\Lambda)=A_{\phi\left(\mathscr{C}_{W}\right)}(\Lambda(W))=\mathrm{I}_{\phi\left(\mathscr{C}_{W}\right)}(\Lambda),
$$

the ambient g.r.s. being $\Phi(W)$ rather than $\Phi$ (but taken in all of $V$ ). Hence: Corollary 2.2 (Arthur). Under the preceding hypotheses,

$$
\amalg_{\phi \bullet \psi}=\sum_{W} \sum_{\mathscr{C}_{W}} \amalg_{\phi\left(\mathscr{C}_{W}\right)} \cdot A_{\psi, \mathscr{E}_{W}} .
$$

There is also a little side issue that might just as well be dealt with here.

Lemma 2.3. If $\phi$ and all its derivatives are slowly increasing, then the same is true of $A_{\phi}$.

Proof. To begin with, let $B$ stand for the closed unit ball in $\sqrt{-1} V$. Then, taking into account Proposition 2.12 of [8], the assignment

$$
\left\{\begin{array}{c}
C^{\infty}(B) \rightarrow C^{\infty}(B) \\
? \rightarrow A_{?}
\end{array}\right.
$$

is continuous (closed graph theorem). That being the case, set $\phi_{t}(\Lambda)=\phi(t \Lambda)$. Obviously,

$$
A_{\phi}(\Lambda)=t^{-l} A_{\phi_{t}}\left(t^{-1} \Lambda\right) .
$$

Assume now that $\|\Lambda\| \leq M$. Then

$$
\begin{aligned}
\left|D A_{\phi}(\Lambda)\right| & \leq M^{l} \bullet\left|D A_{\phi_{M^{-1}}}(M ?)(\Lambda)\right| \\
& \leq M^{k} \bullet\left|D A_{\phi_{M^{-1}}}(M \Lambda)\right| \quad(\exists k) \\
& \leq M^{k} \bullet \rho\left(\phi_{M^{-1}}\right) \quad\left(\rho \text { a seminorm on } C^{\infty}(B)\right) \\
& \leq M^{k^{\prime}} \bullet \sup _{\left\|\Lambda^{\prime}\right\| \leq M}\left|D^{\prime} \phi\left(\Lambda^{\prime}\right)\right| \quad\left(\exists k^{\prime}\right) \\
& \leq M^{k^{\prime \prime}}\left(\exists k^{\prime \prime}\right),
\end{aligned}
$$

finishing the proof. 


\section{ARThUR's manipulation}

The purpose of this section is to formulate an important result due to Arthur [4] that will be applied in the next section during the course of the cancellation. The context within which we wish to work is $\S 9$ of [11]. Although it will be necessary to change what was said there, we shall not insist upon all the details since no really new ideas are involved.

The starting point of the analysis in [11, $\S]$ is the $\bmod o(\mathbf{H})$ expression for

$$
\begin{aligned}
& \left\langle\sum_{m, n} \mathbf{C}_{m n}\left(\alpha: \mathscr{O}_{i_{0}}, \Lambda_{i}+\Lambda_{i_{0}}^{\dagger}\right)_{\mathscr{C}_{i}},\right. \\
& \quad \times\left(\mathbf{Q}_{\mathscr{C}_{i}}^{\mathbf{H}} \mathbf{E}\left(\mathscr{C}_{i}: P_{i_{0}} \mid A_{i_{0}}: \mathbf{e}_{m}^{i_{0}}: \Lambda_{i_{0}}^{\dagger}: ?\right), \mathbf{Q}_{\mathscr{C}_{i}}^{\mathbf{H}} \mathbf{E}\left(\mathscr{C}_{i}: P_{i_{0}} \mid A_{i_{0}}: \mathbf{e}_{n}^{i_{0}}: \Lambda_{i_{0}}^{\dagger}: ?\right)_{\mathscr{C}_{i}}\right\rangle_{\mathscr{C}_{i}}
\end{aligned}
$$

involving derivatives that come up from the evaluation of a Ш-transform at 0 . This time, it will be best for us to use initially a less precise result, saving the refinements for later. Namely, up to $o(\mathbf{H})$, the expression in question is equal to $(-1)^{l_{0}^{\dagger}}$ times the sum

$$
\begin{aligned}
\sum_{w_{i_{0}}^{\dagger}} \operatorname{tr}_{\mathscr{C}_{i}}\left(\amalg_{\phi \bullet \psi}\left(\left(1-w_{i_{0}}^{\dagger}\right) \Lambda_{i_{0}}^{\dagger}\right) \times \operatorname{Ind}_{\mathscr{C}_{i_{0}}}^{G}\left(\left(\mathcal{O}_{i_{0}}, \Lambda_{i}+\Lambda_{i_{0}}^{\dagger}\right)\right)(\alpha)\right. \\
\left.\qquad \mathbf{c}\left(P_{i_{0}}\left|A_{i_{0}}: P_{i_{0}}\right| A_{i_{0}}: w_{i_{0}}^{\dagger}: \Lambda_{i_{0}}^{\dagger}\right)^{*}\right),
\end{aligned}
$$

where $\phi$ and $\psi$ are families of operators on $\mathscr{E}\left(\delta, \mathscr{O}_{i_{0}}\right)$ given as follows: When

$$
\begin{aligned}
& \mathscr{C}^{\dagger} \leftrightarrow\left(j_{0}, w_{j_{0} i_{0}}^{\dagger}\right), \\
& \phi_{\mathscr{E}^{\dagger}}(\Lambda)= \mathbf{c}\left(P_{j_{0}}\left|A_{j_{0}}: P_{i_{0}}\right| A_{i_{0}}: w_{j_{0} i_{0}}^{\dagger}: w_{i_{0}}^{\dagger} \Lambda_{i_{0}}^{\dagger}\right)^{*} \\
& \bullet \mathbf{e}\left(\mathscr{C}_{j_{0}}: \mathbf{H}: w_{j_{0} i_{0}}^{\dagger} \Lambda\right) \\
& \bullet \mathbf{c}\left(P_{j_{0}}\left|A_{j_{0}}: P_{i_{0}}\right| A_{i_{0}}: w_{j_{0} i_{0}}^{\dagger}: w_{i_{0}}^{\dagger} \Lambda_{i_{0}}^{\dagger}\right),
\end{aligned}
$$

while

$$
\begin{aligned}
\psi_{\mathscr{C}^{\dagger}}(\Lambda)= & \mathbf{c}\left(P_{j_{0}}\left|A_{j_{0}}: P_{i_{0}}\right| A_{i_{0}}: w_{j_{0} i_{0}}^{\dagger}: w_{i_{0}}^{\dagger} \Lambda_{i_{0}}^{\dagger}\right)^{-1} \\
& \bullet \mathbf{c}\left(P_{j_{0}}\left|A_{j_{0}}: P_{i_{0}}\right| A_{i_{0}}: w_{j_{0} i_{0}}^{\dagger}: \Lambda-w_{i_{0}}^{\dagger} \overline{\Lambda_{i_{0}}^{\dagger}}\right) .
\end{aligned}
$$

We explicitly observe that $\phi$ and $\psi$ depend on $\Lambda_{i_{0}}^{\dagger}$. Individually, however, neither $\phi$ nor $\psi$ need be a Detroit family although their product $\phi \bullet \psi$ is.

The motivation for these choices (among the many possible) can be found in the discussion centering on Lemma 9.5 of [11]. Even so, it will be best to modify them a little. To see why, pretend for the moment that there is just one cusp; then the c-functions inherent in the definition of $\phi$ drop out giving an exponential (which is a pure scalar). Since this is not the case in general, we shall employ the following artifice. Fix $\mathbf{H}_{00} \in \mathfrak{a}$ and let

$$
\mathbf{H}=\mathbf{H}_{00}+\mathbf{H}_{0} \quad\left(\mathbf{H}_{0} \in \mathfrak{a}_{0}(\cdot)\right),
$$


the idea being that $\mathbf{H}_{0}$ will eventually be sent to $-\infty$ in the $\lim _{0}$-sense (cf. [8, p. 171]). With, as before, $\mathscr{C}^{\dagger} \leftrightarrow\left(j_{0}, w_{j_{0} i_{0}}^{\dagger}\right)$, put

$$
\phi_{\mathscr{C}^{\dagger}}(\Lambda)=\exp \left(\left\langle\mathbf{H}_{0}\left(P_{j_{0}}\right), w_{j_{0} i_{0}}^{\dagger} \Lambda\right\rangle\right)
$$

and

$$
\begin{aligned}
\psi_{\mathscr{B}^{\dagger}}(\Lambda)= & \mathbf{c}\left(P_{j_{0}}\left|A_{j_{0}}: P_{i_{0}}\right| A_{i_{0}}: w_{j_{0} i_{0}}^{\dagger} w_{i_{0}}^{\dagger}: \Lambda_{i_{0}}^{\dagger}\right)^{*} \\
& \bullet \mathbf{e}\left(\mathscr{C}_{j_{0}}: \mathbf{H}_{00}: w_{j_{0} i_{0}}^{\dagger} \Lambda\right) \\
& \bullet \mathbf{c}\left(P_{j_{0}}\left|A_{j_{0}}: P_{i_{0}}\right| A_{i_{0}}: w_{j_{0} i_{0}}^{\dagger}: \Lambda-w_{i_{0}}^{\dagger} \overline{\Lambda_{i_{0}}^{\dagger}}\right) .
\end{aligned}
$$

In this connection, observe that the functional equations imply

$$
\begin{aligned}
\mathbf{c}\left(P_{i_{0}}\left|A_{i_{0}}: P_{i_{0}}\right| A_{i_{0}}: w_{i_{0}}^{\dagger}: \Lambda_{i_{0}}^{\dagger}\right)^{*} \bullet \mathbf{c}\left(P_{j_{0}}\left|A_{j_{0}}: P_{i_{0}}\right| A_{i_{0}}: w_{j_{0} i_{0}}^{\dagger}: w_{i_{0}}^{\dagger} \Lambda_{i_{0}}^{\dagger}\right)^{*} \\
=\left[\mathbf{c}\left(P_{j_{0}}\left|A_{j_{0}}: P_{i_{0}}\right| A_{i_{0}}: w_{j_{0} i_{0}}^{\dagger}: w_{i_{0}}^{\dagger} \Lambda_{i_{0}}^{\dagger}\right) \bullet \mathbf{c}\left(P_{i_{0}}\left|A_{i_{0}}: P_{i_{0}}\right| A_{i_{0}}: w_{i_{0}}^{\dagger}: \Lambda_{i_{0}}^{\dagger}\right)\right]^{*} \\
=\mathbf{c}\left(P_{j_{0}}\left|A_{j_{0}}: P_{i_{0}}\right| A_{i_{0}}: w_{j_{0} i_{0}}^{\dagger} w_{i_{0}}^{\dagger}: \Lambda_{i_{0}}^{\dagger}\right)^{*},
\end{aligned}
$$

thus we are incorporating

$$
\mathbf{c}\left(P_{i_{0}}\left|A_{i_{0}}: P_{i_{0}}\right| A_{i_{0}}: w_{i_{0}}^{\dagger}: \Lambda_{i_{0}}^{\dagger}\right)^{*}
$$

as part of the new definition of $\psi$. On the basis of this revision, the expression that we want to examine is equal to $(-1)^{l_{0}^{\dagger}}$ times the sum

$$
\sum_{w_{i_{0}}^{\dagger}} \mathbf{t r}_{\mathscr{C}_{i}}\left(\mathrm{II}_{\phi \bullet \psi}\left(\left(1-w_{i_{0}}^{\dagger}\right) \Lambda_{i_{0}}^{\dagger}\right) \bullet \mathbf{I n d}_{\mathscr{\delta}_{i_{0}}}^{G}\left(\left(\mathscr{O}_{i_{0}}, \Lambda_{i}+\Lambda_{i_{0}}^{\dagger}\right)\right)(\alpha)\right) .
$$

Noting that $\phi$ and $\psi$ are both Detroit families, the next step is to use Corollary 2.2 and write

$$
\mathrm{\amalg}_{\phi \bullet \psi}=\sum_{W} \sum_{\mathscr{E}_{W}} \amalg_{\phi\left(\mathscr{B}_{W}\right)} \bullet A_{\psi, \mathscr{C}_{W}},
$$

then integrate according to the scheme

$$
\int_{\operatorname{Re}\left(\Lambda_{i}\right)=0} \int_{\operatorname{Re}\left(\Lambda_{i_{0}}^{\dagger}\right)=0}=\int_{\operatorname{Re}\left(\Lambda_{i_{0}}\right)=0},
$$

pulling, as is permissible, the sum over the $w_{i_{0}}^{\dagger}$ to the outside: In other words, look at

$$
\sum_{w_{i_{0}}^{\dagger}} \sum_{W} \sum_{\mathscr{C}_{W}} \int_{\operatorname{Re}\left(\Lambda_{i_{0}}\right)=0} \cdots\left|d \Lambda_{i_{0}}\right|,
$$

where the $\cdots$ denotes

$$
\mathrm{W}_{\phi\left(\mathscr{E}_{W}\right)}\left(\left(1-w_{i_{0}}^{\dagger}\right) \Lambda_{i_{0}}^{\dagger}\right) \mathbf{t r}_{\mathscr{C}_{i}}\left(A_{\psi}, \mathscr{C}_{W}\left(\left(1-w_{i_{0}}^{\dagger}\right) \Lambda_{i_{0}}^{\dagger}\right) \bullet \operatorname{Ind}_{\mathscr{C}_{i_{0}}}^{G}\left(\left(\mathscr{O}_{i_{0}}, \Lambda_{i_{0}}\right)\right)(\alpha)\right) .
$$


Fix $W$ and $\mathscr{C}_{W}$ and let $f$ be a representative component of $\operatorname{tr}_{\mathscr{C}_{i}}($ ?). Then $f$ is Schwartz (cf. Lemma 9.3 of [11] and Lemma 2.3) and a typical term in our sum is

$$
\int_{\operatorname{Re}\left(\Lambda_{i_{0}}\right)=0} \amalg_{\phi\left(\mathscr{C}_{W}\right)}\left(\left(1-w_{i_{0}}^{\dagger}\right) \Lambda_{i_{0}}^{\dagger}\right) f\left(\Lambda_{i_{0}}\right)\left|d \Lambda_{i_{0}}\right| .
$$

To make the presence of $w_{i_{0}}^{\dagger}$ felt, let $W_{0}=\operatorname{Im}\left(1-w_{i_{0}}^{\dagger}\right)$ be a special subspace (see the Appendix to this section), and define

$$
F(\Lambda)=\int_{\sqrt{-1} \operatorname{Ker}\left(1-w_{i_{0}}^{\dagger}\right)} f\left(\Lambda+\Lambda_{i_{0}}\right)\left|d \Lambda_{i_{0}}\right| .
$$

Then $F$ is still Schwartz and what remains is

$$
\frac{1}{\left|\operatorname{det}\left(\left(1-w_{i_{0}}^{\dagger}\right) \mid \operatorname{Ker}\left(1-w_{i_{0}}^{\dagger}\right)^{\perp}\right)\right|} \int_{\sqrt{-1} W_{0}} \amalg_{\phi\left(\mathscr{C}_{W}\right)}(\Lambda) F(\Lambda)|d \Lambda| .
$$

The integral over $\sqrt{-1} W_{0}$ is a function of $\mathbf{H}$ and the central question is: How does it behave as $\mathbf{H}_{0} \rightarrow-\infty$ in the $\lim _{0}$-sense? The following is the answer.

Theorem 3.1. The integral $\int_{\sqrt{-1} W_{0}} \amalg_{\phi\left(\mathscr{C}_{W}\right)}(\Lambda) F(\Lambda)|d \Lambda|$ is equal to a rapidly decreasing function of $\mathbf{H}$ plus 0 if $W_{0} \not \subset W$ or $(-2 \pi)^{\operatorname{dim}\left(W_{0}\right)} \bullet \amalg_{\phi\left(\mathscr{C}_{W}\right)}^{W_{0}}(0) F(0)$ if $W_{0} \subset W$.

[Note: $\Psi_{\phi\left(\mathscr{E}_{W}\right)}^{W_{0}}$ is the $\amalg$-transform of the object manufactured from $\phi$ by descent to $W_{0}^{\perp} \cap W$.]

This result is implicit in Arthur [4, §4]. Because he does not quite state it this way and because our conventions are not the same as his, we shall give a brief indication of what is involved in the proof.

To simplify the notation, we might just as well suppose that $\mathfrak{a}_{i_{0}}=\mathfrak{a}_{i_{0}}^{\dagger}$. In addition, if

$$
\mathbf{H}_{\mathscr{C}} \equiv \mathbf{H}_{0}\left(P_{\mathscr{C}}\right) \text {, }
$$

where $P_{\mathscr{C}}$ is the split parabolic subgroup of $G$ having $\mathscr{C}$ for its positive chamber (cf. TES, p. 66), then we can and will assume that $\mathbf{H}_{\mathscr{E}} \in-\mathscr{C}$.

Now call $\operatorname{Con}(\mathbf{H})$ the convex hull of the $\mathbf{H}_{\mathscr{C}}$. It is well known that

$$
H \in(-\mathscr{C}) \cap \operatorname{Con}(\mathbf{H}) \Leftrightarrow \Gamma_{*}^{F_{0}(\mathscr{C})}\left(H, \mathbf{H}_{\mathscr{C}}\right) \neq 0
$$

(see, e.g., Lemma 3.2 of [1]). Consequently,

$$
\left.\sum_{\mathscr{C}} \Gamma_{*}^{F_{0}^{(\mathscr{C})}}\left(?, \mathbf{H}_{\mathscr{C}}\right)=\chi_{\mathbf{H}} \quad \text { (a.e. }\right),
$$

where $\chi_{\mathbf{H}}$ is the characteristic function of $\operatorname{Con}(\mathbf{H})$. This implies that

$$
\amalg_{\phi}=(-1)^{l_{0}} \hat{\chi}_{\mathbf{H}} \text {. }
$$

In general, if $\mathscr{C} \leftrightarrow\left(\mathscr{C}_{W}, \mathscr{C}(W)\right)$, then analogously

$$
\amalg_{\phi\left(\mathscr{C}_{W}\right)}=(-1)^{\operatorname{dim}(W)} \bullet \exp \left(\left\langle\mathbf{H}_{\mathscr{C}_{W}}^{\dagger}, ?\right\rangle\right) \hat{\chi}_{\mathbf{H}\left(\mathscr{C}_{W}\right)},
$$


where, of course, $\mathbf{H}_{\mathscr{C}_{W}}^{\dagger}$ is the projection of $\mathbf{H}_{\mathscr{C}}$ onto $W^{\perp}$. Introducing

$$
\widehat{F}_{0}(H)=\int_{\sqrt{-1} W_{0}} F(\Lambda) e^{(\Lambda, H)}|d \Lambda|,
$$

a function on $\mathfrak{a}_{i_{0}}$ that is Schwartz on $W_{0}$ and invariant under $W_{0}^{\perp}$, we conclude that

$$
\begin{aligned}
& \int_{\sqrt{-1} W_{0}} \amalg_{\phi\left(\mathscr{C}_{W}\right)}(\Lambda) F(\Lambda)|d \Lambda| \\
& \quad=(-1)^{\operatorname{dim}(W)} \bullet \int_{\operatorname{Con}\left(\mathbf{H}\left(\mathscr{C}_{W}\right)\right)} \widehat{F}_{0}\left(H+\mathbf{H}_{\mathscr{C}_{W}}^{\dagger}\right) d H .
\end{aligned}
$$

Case I. $W_{0} \not \subset W$. In this situation,

$$
\int_{\operatorname{Con}\left(\mathbf{H}\left(\mathscr{E}_{W}\right)\right)} \widehat{F}_{0}\left(H+\mathbf{H}_{\mathscr{C}_{W}}^{\dagger}\right) d H
$$

is a rapidly decreasing function of $\mathbf{H}$. The proof can be extracted from the discussion on p. 1306 of [4]. Roughly speaking, the presence of $\mathbf{H}_{\mathscr{C}_{W}}^{\dagger}$ forces the integrand to decrease rapidly. But matters can simultaneously be arranged so as to ensure that the measure of the region of integration increases slowly. The geometric tools needed to pin these points down precisely are in [4, §3].

Case II. $W_{0} \subset W$. In this situation,

$$
\int_{\operatorname{Con}\left(\mathbf{H}\left(\mathscr{C}_{W}\right)\right)} \widehat{F}_{0}\left(H+\mathbf{H}_{\mathscr{C}_{W}}^{\dagger}\right) d H=\int_{\operatorname{Con}\left(\mathbf{H}\left(\mathscr{C}_{W}\right)\right)} \widehat{F}_{0}(H) d H,
$$

the integrand being invariant under $W_{0}^{\perp}$ and

$$
\left\{\begin{array}{l}
W_{0} \subset W \Rightarrow W^{\perp} \subset W_{0}^{\perp}, \\
\mathbf{H}_{\mathscr{C}_{W}}^{\dagger} \in W^{\perp} .
\end{array}\right.
$$

To keep the notation simple, take $W=\mathfrak{a}_{i_{0}}$. Put

$$
K_{0}=\bigcap_{\mathscr{C}_{W_{0}}} \operatorname{Con}\left(\left\{\mathbf{H}_{\mathscr{C}}-\mathbf{H}_{\mathscr{C}_{W_{0}}}^{\dagger}: \forall \mathscr{C}\left(W_{0}\right)\right\}\right) .
$$

Then, using ideas from $\S 3$ of [4], one can show that

$$
\operatorname{Con}(\mathbf{H}) \cap\left(K_{0} \times W_{0}^{\perp}\right)=K_{0} \times \operatorname{Con}\left(\mathbf{H}^{\dagger}\right) .
$$

Accordingly,

$$
\begin{aligned}
\int_{\operatorname{Con}(\mathbf{H})} & \widehat{F}_{0}(H) d H=\int_{\operatorname{Con}(\mathbf{H})-K_{0} \times \operatorname{Con}\left(\mathbf{H}^{\dagger}\right)} \widehat{F}_{0}(H) d H+\int_{K_{0} \times \operatorname{Con}\left(\mathbf{H}^{\dagger}\right)} \widehat{F}_{0}(H) d H \\
= & \int_{\operatorname{Con}(\mathbf{H})-K_{0} \times \operatorname{Con}\left(\mathbf{H}^{\dagger}\right)} \widehat{F}_{0}(H) d H+\operatorname{vol}\left(\operatorname{Con}\left(\mathbf{H}^{\dagger}\right)\right) \bullet \int_{K_{0}} \widehat{F}_{0}(H) d H \\
= & \int_{\operatorname{Con}(\mathbf{H})-K_{0} \times \operatorname{Con}\left(\mathbf{H}^{\dagger}\right)} \widehat{F}_{0}(H) d H-\operatorname{vol}\left(\operatorname{Con}\left(\mathbf{H}^{\dagger}\right)\right) \bullet \int_{W_{0}-K_{0}} \widehat{F}_{0}(H) d H \\
& +\operatorname{vol}\left(\operatorname{Con}\left(\mathbf{H}^{\dagger}\right)\right) \bullet \int_{W_{0}} \widehat{F}_{0}(H) d H .
\end{aligned}
$$


Terms one and two are rapidly decreasing functions of $\mathbf{H}$. Turning to the third term, we have, from the definitions,

$$
\operatorname{vol}\left(\operatorname{Con}\left(\mathbf{H}^{\dagger}\right)\right)=(-1)^{l_{0}-\operatorname{dim}\left(W_{0}\right)} \bullet \Psi_{\phi}^{W_{0}}(0),
$$

while, by Fourier inversion,

$$
\int_{W_{0}} \widehat{F}_{0}(H) d H=(2 \pi)^{\operatorname{dim}\left(W_{0}\right)} \bullet F(0) .
$$

The $(-1)^{l_{0}}$ that cropped up when we started cancels the $(-1)^{l_{0}}$ appearing above (remember that the ambient special subspace is, momentarily, $\mathfrak{a}_{i_{0}}$ itself). This does it.

Now let us go back and apply this result. Because $1-w_{i_{0}}^{\dagger}$ is normal,

$$
\operatorname{Ker}\left(1-w_{i_{0}}^{\dagger}\right)^{\perp}=\operatorname{Im}\left(1-w_{i_{0}}^{\dagger}\right) .
$$

Ignoring anything that is $\mathbf{o}(\mathbf{H})$, we are effectively left with

$$
\begin{aligned}
& (-2 \pi)^{\operatorname{rank}\left(1-w_{i_{0}}^{\dagger}\right)} \bullet \sum_{W, \mathscr{C}_{W}: \operatorname{Im}\left(1-w_{i_{0}}^{\dagger}\right) \subset W} \amalg_{\phi\left(\mathscr{C}_{W}\right)}^{\operatorname{Im}\left(1-w_{i_{0}}^{\dagger}\right)}(0) \\
& \times \int_{\sqrt{-1} \operatorname{Ker}\left(1-w_{i_{0}}^{\dagger}\right)} \mathbf{t r}_{\mathscr{C}_{i}}\left(A_{\psi}, \mathscr{C}_{W}(0) \bullet \operatorname{Ind}_{\mathscr{E}_{i_{0}}}^{G}\left(\left(\mathscr{O}_{i_{0}}, \Lambda_{i_{0}}\right)\right)(\alpha)\right)\left|d \Lambda_{i_{0}}\right| .
\end{aligned}
$$

This can be reassembled. Thus introduce a Detroit family $\boldsymbol{\theta}_{i_{0}}$ by the prescription

$$
\begin{aligned}
{\left[\theta_{i_{0}}\right]_{\mathscr{G}^{\dagger}}(\Lambda)=} & \mathbf{c}\left(P_{j_{0}}\left|A_{j_{0}}: P_{i_{0}}\right| A_{i_{0}}: w_{j_{0} i_{0}}^{\dagger} w_{i_{0}}^{\dagger}: \Lambda_{i_{0}}\right)^{*} \\
& \bullet \mathbf{e}\left(\mathscr{C}_{j_{0}}: \mathbf{H}: w_{j_{0} i_{0}}^{\dagger} \Lambda\right) \\
& \bullet \mathbf{c}\left(P_{j_{0}}\left|A_{j_{0}}: P_{i_{0}}\right| A_{i_{0}}: w_{j_{0} i_{0}}^{\dagger}: \Lambda-w_{i_{0}}^{\dagger} \bar{\Lambda}_{i_{0}}\right),
\end{aligned}
$$

where, as usual, $\mathscr{C}^{\dagger} \leftrightarrow\left(j_{0}, w_{j_{0} i_{0}}^{\dagger}\right)$. Denote by $\amalg_{\boldsymbol{\theta}_{i_{0}}}^{w_{i_{0}}^{\dagger}}$ its $\amalg$-transform (per $\left.\operatorname{Ker}\left(1-w_{i_{0}}^{\dagger}\right)\right)$. The foregoing then becomes

$$
(-2 \pi)^{\operatorname{rank}\left(1-w_{i_{0}}^{\dagger}\right)} \int_{\sqrt{-1} \operatorname{Ker}\left(1-w_{i_{0}}^{\dagger}\right)} \operatorname{tr}_{\mathscr{E}_{i}}\left(\boldsymbol{W}_{\boldsymbol{\theta}_{i_{0}}}^{w_{i_{0}}^{\dagger}}(0) \bullet \operatorname{Ind}_{\mathscr{C}_{i_{0}}}^{G}\left(\left(\mathscr{O}_{i_{0}}, \Lambda_{i_{0}}\right)\right)(\alpha)\right)\left|d \Lambda_{i_{0}}\right| .
$$

Lest we forget, there is a $(-1)^{l_{0}^{\dagger}}$ lurking in the background, as well as the factor

$$
\frac{1}{\left|\operatorname{det}\left(\left(1-w_{i_{0}}^{\dagger}\right) \mid \operatorname{Im}\left(1-w_{i_{0}}^{\dagger}\right)\right)\right|} .
$$

Appendix. There is a technicality, sometimes used tacitly, that probably should not be swept under the rug entirely, viz: 
Lemma. Let $P_{i_{0}}$ be a cuspidal split parabolic subgroup of $G$ with split component $A_{i_{0}}$; let $w_{i_{0}} \in W\left(A_{i_{0}}\right)$. Then $\exists w_{j_{0} i_{0}} \in W\left(A_{j_{0}}, A_{i_{0}}\right)$ such that

$$
w_{j_{0}}=w_{j_{0} i_{0}} w_{i_{0}} w_{j_{0} i_{0}}^{-1}
$$

has the property that $\operatorname{Ker}\left(1-w_{j_{0}}\right)$ is the split component of $a P_{j} \succeq P_{j_{0}}$, the association class of $P_{j}$ being uniquely determined.

[Note: It therefore follows that $\operatorname{Im}\left(1-w_{i_{0}}\right)$ is special.]

The uniqueness is easy. Indeed, if $w_{j_{0} i_{0}} \in W\left(A_{j_{0}}, A_{i_{0}}\right)$ and $w_{k_{0} i_{0}} \in$ $W\left(A_{k_{0}}, A_{i_{0}}\right)$ are as above, then

$$
w_{k_{0}}=w_{k_{0} j_{0}} w_{j_{0}} w_{k_{0} j_{0}}^{-1} \quad\left(\exists w_{k_{0} j_{0}} \in W\left(A_{k_{0}}, A_{j_{0}}\right)\right)
$$

with $\operatorname{Ker}\left(1-w_{j_{0}}\right)=\mathfrak{a}_{j}, \operatorname{Ker}\left(1-w_{k_{0}}\right)=\mathfrak{a}_{k}$, and $w_{k_{0} j_{0}}\left(\mathfrak{a}_{j}\right)=\mathfrak{a}_{k}$, so $P_{j}$ and $P_{k}$ are associate.

Passing to the existence, the strategy will be to reduce it to the percuspidal case and then apply a generality. Suppose that we knew that there was a subset $F_{i_{0}}$ of $\Sigma_{P_{i_{0}}}\left(\mathfrak{g}, \mathfrak{a}_{i_{0}}\right)$ (cf. TES, p. 32) such that

$$
\left(\mathfrak{a}_{i_{0}}\right)_{F_{i_{0}}}=\operatorname{Ker}\left(1-w_{i_{0}}\right) .
$$

Then there would be a chamber set $F$ in $\Sigma_{P_{i_{0}}}\left(\mathfrak{g}, \mathfrak{a}_{i_{0}}\right)$ such that

$$
\left(\mathfrak{a}_{i_{0}}\right)_{F}=\operatorname{Ker}\left(1-w_{i_{0}}\right) \text {. }
$$

For this, see [8, pp. 151-152]. By definition, $F \subset F_{0}\left(\mathscr{C}_{0}\right), \mathscr{C}_{0}$ a chamber, hence there exists a $w_{j_{0} i_{0}} \in W\left(A_{j_{0}}, A_{i_{0}}\right)$ such that

$$
w_{j_{0} i_{0}}\left(\mathscr{C}_{0}\right)=\mathscr{C}_{P_{j_{0}}}\left(\mathfrak{a}_{j_{0}}\right) \text {. }
$$

With $w_{j_{0}}$ defined per supra,

$$
\operatorname{Ker}\left(1-w_{j_{0}}\right)=\left(\mathfrak{a}_{j_{0}}\right)_{w_{j_{0} i_{0}}(F)},
$$

the sought for $\mathfrak{a}_{j}$. Now pick a percuspidal $P_{0} \preceq P_{i_{0}}$. Extend, as is possible, $w_{i_{0}}$ to an element $w_{0} \in W\left(A_{0}\right)$ (cf. TES, p. 68). Suppose that we knew that there was a subset $F_{0}$ of $\Sigma_{P_{0}}\left(\mathfrak{g}, \mathfrak{a}_{0}\right)$ (cf. TES, p. 32) such that $\left(\mathfrak{a}_{0}\right)_{F_{0}}=\operatorname{Ker}\left(1-w_{0}\right)$. Put

$$
F_{i_{0}}=\left\{\lambda \mid \mathfrak{a}_{i_{0}}: \lambda \in F_{0}\right\} \subset \Sigma_{P_{i_{0}}}\left(\mathfrak{g}, \mathfrak{a}_{i_{0}}\right) .
$$

Then

$$
\left(\mathfrak{a}_{i_{0}}\right)_{F_{i_{0}}}=\operatorname{Ker}\left(1-w_{i_{0}}\right),
$$

implying that there is no loss of generality in assuming that $P_{i_{0}}$ is percuspidal.

But this simplification allows one to work directly within the context of an abstract root system and its Weyl group. Consequently, it will be enough to establish: 
Sublemma. Let $\Phi$ be a root system in a vector space $V$ and let $w$ be an element of its Weyl group. Then $\operatorname{Im}(1-w)$ is spanned by its intersection with $\Phi$.

[This, no doubt, is well known. The point is that if $H$ is a nonzero element of $V$ which is fixed by $w$, then $w$ is a product of simple reflections fixing $H$. From them, one can construct a subset of $\Phi$, orthogonal to $H$, that is a root system whose Weyl group contains $w$ and then argue by induction on the dimension of its span.]

\section{Cancellation}

The purpose of this section is to carry out the cancellation, the technical basis for our approach to deriving the contribution to the trace arising from the continuous spectrum (cf. $\S 5$ infra). Thus, agreeing to subscribe to the assumptions and conventions of $[11, \S 9]$, let $\alpha$ be a $K$-central, $K$-finite element of $C_{c}^{\infty}(G)$. Then $L_{G / \Gamma}^{\mathrm{dis}}(\alpha)$ is of the trace class with

$$
\operatorname{tr}\left(L_{G / \Gamma}^{\mathrm{dis}}(\alpha)\right)=\lim _{\mathbf{H} \rightarrow-\infty} \operatorname{tr}\left(Q^{\mathbf{H}} \circ L_{G / \Gamma}^{\mathrm{dis}}(\alpha) \circ Q^{\mathbf{H}}\right) .
$$

And, applying what was learned in $\S 3 \bmod o(\mathbf{H})$,

$$
\operatorname{tr}\left(Q^{\mathbf{H}} \circ L_{G / \Gamma}^{\mathrm{dis}}(\alpha) \circ Q^{\mathbf{H}}\right)
$$

is the same as $\mathbf{K}\left(\mathbf{H}_{\mathrm{O}}: \alpha: \Gamma\right)$ less

$$
\sum_{\mathscr{C}_{i}, \mathscr{C}_{i_{0}}} \sum_{\mathscr{O}_{i_{0}}} \int_{\operatorname{Re}\left(\Lambda_{i_{0}}\right)=0}\left\langle\mathbf{p}\left(\Gamma: \mathscr{C}_{i}: \mathbf{H}-\mathbf{H}_{\mathrm{O}}\right), \mathbf{o}_{\mathscr{C}_{i}}\left(\mathscr{O}_{i_{0}}, \Lambda_{i_{0}}: \mathbf{H}\right)\right\rangle\left|d \Lambda_{i_{0}}\right|
$$

less

$$
\begin{aligned}
& \sum_{\mathscr{E}_{i}, \mathscr{E}_{i_{0}}} \sum_{\mathscr{O}_{i_{0}}}(-1)^{\mathrm{rank}\left(\mathscr{E}_{i}\right)} \cdot \frac{1}{*\left(\mathscr{C}_{i_{0}}^{\dagger}\right)} \cdot \frac{1}{(2 \pi)^{l_{0}}} \\
& \text { - }\left\langle\mathbf{p}\left(\Gamma: \mathscr{C}_{i}: \mathbf{H}-\mathbf{H}_{\mathrm{O}}\right),(-1)^{l_{0}^{\dagger}}\right. \\
& \text { - } \sum_{w_{i_{0}}^{\dagger}}(-2 \pi)^{\operatorname{rank}\left(1-w_{i_{0}}^{\dagger}\right)} \cdot \frac{1}{\left|\operatorname{det}\left(\left(1-w_{i_{0}}^{\dagger}\right) \mid \operatorname{Im}\left(1-w_{i_{0}}^{\dagger}\right)\right)\right|} \\
& \left.\times \int_{\sqrt{-1} \operatorname{Ker}\left(1-w_{i_{0}}^{\dagger}\right)} \mathbf{t r}_{\mathscr{E}_{i}}\left(\amalg_{\boldsymbol{\theta}_{i_{0}}}^{w_{i_{0}}^{\dagger}}(0) \bullet \mathbf{I n d}_{\mathscr{C}_{i_{0}}}^{G}\left(\left(\mathscr{O}_{i_{0}}, \Lambda_{i_{0}}\right)\right)(\alpha)\right)\right\rangle\left|d \Lambda_{i_{0}}\right|,
\end{aligned}
$$

where the sum $\sum_{\mathscr{C}_{i}, \mathscr{C}_{i_{0}}}$ extends over all $\mathscr{C}_{i}, \mathscr{C}_{i_{0}}$ such that $\mathscr{C}_{i} \succeq \mathscr{C}_{i_{0}}\left(\mathscr{C}_{i_{0}} \neq\{G\}\right)$. Here, $\mathbf{K}\left(\mathbf{H}_{\mathrm{O}}: \alpha: \Gamma\right)$ contains the contribution to the trace associated with the conjugacy classes, a subject that will be taken up in another place. On the other hand, in $\S 6$ infra, it will be shown that

$$
\sum_{\mathscr{C}_{i}, \mathscr{C}_{i_{0}}} \sum_{\mathscr{O}_{i_{0}}} \int_{\operatorname{Re}\left(\Lambda_{i_{0}}\right)=0}\left\langle\mathbf{p}\left(\Gamma: \mathscr{C}_{i}: \mathbf{H}-\mathbf{H}_{\mathrm{O}}\right), \mathbf{o}_{\mathscr{C}_{i}}\left(\mathscr{O}_{i_{0}}, \Lambda_{i_{0}}: \mathbf{H}\right)\right\rangle\left|d \Lambda_{i_{0}}\right|
$$


is $\mathbf{o}(\mathbf{H})$, so in the end is of no concern. We shall therefore direct our attention to the final term, call it $\mathbf{F n c}\left(\mathbf{H}: \mathbf{H}_{\mathrm{O}}: \alpha: \Gamma\right)$, in which, as we shall eventually see, the contribution to the trace arising from the continuous spectrum is to be found.

Before proceeding, it will be best to make a couple of adjustments in the signs. By definition, $l_{i}=\operatorname{rank}\left(\mathscr{C}_{i}\right)$ and $l_{0}=l_{i}+l_{0}^{\dagger}$. Therefore $\operatorname{Fnc}\left(\mathbf{H}: \mathbf{H}_{\mathrm{O}}: \alpha: \Gamma\right)$ is equal to

$$
\begin{aligned}
& \sum_{\mathscr{E}_{i}, \mathscr{E}_{i_{0}}} \sum_{\mathscr{O}_{i_{0}}} \frac{1}{*\left(\mathscr{C}_{i_{0}}^{\dagger}\right)} \bullet\left\langle\mathbf{p}\left(\Gamma: \mathscr{C}_{i}: \mathbf{H}-\mathbf{H}_{\mathrm{O}}\right),\right. \\
& \sum_{w_{i_{0}}^{\dagger}}\left(-\frac{1}{2 \pi}\right)^{l_{0}-\operatorname{rank}\left(1-w_{i_{0}}^{\dagger}\right)} \bullet \frac{1}{\operatorname{det}\left(\left(1-w_{i_{0}}^{\dagger}\right) \mid \operatorname{Im}\left(1-w_{i_{0}}^{\dagger}\right)\right) \mid} \\
&\left.\times \int_{\sqrt{-1} \operatorname{Ker}\left(1-w_{i_{0}}^{\dagger}\right)} \operatorname{tr}_{\mathscr{C}_{i}}\left(\mathbf{W}_{\boldsymbol{\theta}_{i_{0}}}^{w_{i_{0}}^{\dagger}}(0) \bullet \mathbf{I n d}_{\mathscr{\mathscr { C }}_{i_{0}}}^{G}\left(\left(\mathscr{O}_{i_{0}}, \Lambda_{i_{0}}\right)\right)(\alpha)\right)\right\rangle\left|d \Lambda_{i_{0}}\right| .
\end{aligned}
$$

The analysis of $\int_{\sqrt{-1} \operatorname{Ker}\left(1-w_{i_{0}}^{\dagger}\right)} \cdots$ begins by invoking the Appendix of $\S 3$ to justify a move to an $\mathfrak{a}_{k_{0}}^{\dagger}$ where $\operatorname{Ker}\left(1-w_{k_{0}}^{\dagger}\right.$ ) is a split component (we shall hold the subscript $j_{0}$ in reserve). So, as there, write

$$
w_{k_{0}}^{\dagger}=w_{k_{0} i_{0}}^{\dagger} w_{i_{0}}^{\dagger} w_{k_{0} i_{0}}^{-\dagger}
$$

and set $\Lambda_{k_{0}}=w_{k_{0} i_{0}}^{\dagger} \Lambda_{i_{0}}$. Then

$$
\begin{aligned}
& \operatorname{tr}_{\mathscr{C}_{i}}\left(\amalg_{\boldsymbol{\theta}_{i_{0}}}^{w_{i_{0}}^{\dagger}}(0) \bullet \operatorname{Ind}_{\mathscr{\mathscr { C }}_{i_{0}}}^{G}\left(\left(\mathscr{O}_{i_{0}}, \Lambda_{i_{0}}\right)\right)(\alpha)\right) \\
& =\operatorname{tr}_{\mathscr{E}_{i}}\left(\mathbf{c}\left(P_{k_{0}}\left|A_{k_{0}}: P_{i_{0}}\right| A_{i_{0}}: w_{k_{0} i_{0}}^{\dagger}: \Lambda_{i_{0}}\right) \bullet \amalg_{\boldsymbol{\theta}_{i_{0}}}^{w_{i_{0}}^{\dagger}}(0)\right. \\
& \left.\times \operatorname{Ind}_{\mathscr{\delta}_{i_{0}}}^{G}\left(\left(\mathscr{O}_{i_{0}}, \Lambda_{i_{0}}\right)\right)(\alpha) \bullet \mathbf{c}\left(P_{i_{0}}\left|A_{i_{0}}: P_{k_{0}}\right| A_{k_{0}}: w_{k_{0} i_{0}}^{-\dagger}: \Lambda_{k_{0}}\right)\right) \\
& =\operatorname{tr}_{\mathscr{C}_{i}}\left(\mathbf{c}\left(P_{k_{0}}\left|A_{k_{0}}: P_{i_{0}}\right| A_{i_{0}}: w_{k_{0} i_{0}}^{\dagger}: \Lambda_{i_{0}}\right) \bullet \amalg_{\boldsymbol{\theta}_{i_{0}}}^{w_{i_{0}}^{\dagger}}(0)\right. \\
& \left.\times \mathbf{c}\left(P_{i_{0}}\left|A_{i_{0}}: P_{k_{0}}\right| A_{k_{0}}: w_{k_{0} i_{0}}^{-\dagger}: \Lambda_{k_{0}}\right) \bullet \operatorname{Ind}_{\mathscr{C}_{k_{0}}}^{G}\left(\left(\mathscr{O}_{k_{0}}, \Lambda_{k_{0}}\right)\right)(\alpha)\right) \text {. }
\end{aligned}
$$

But

$$
\begin{aligned}
& \mathbf{c}\left(P_{k_{0}}\left|A_{k_{0}}: P_{i_{0}}\right| A_{i_{0}}: w_{k_{0} i_{0}}^{\dagger}: \Lambda_{i_{0}}\right) \\
& \quad \bullet \amalg_{\boldsymbol{\theta}_{i_{0}}}^{w_{i_{0}}^{\dagger}}(0) \bullet \mathbf{c}\left(P_{i_{0}}\left|A_{i_{0}}: P_{k_{0}}\right| A_{k_{0}}: w_{k_{0} i_{0}}^{-\dagger}: \Lambda_{k_{0}}\right)
\end{aligned}
$$

is equal to the limit as $\Lambda \rightarrow 0$ in $\sqrt{-1} \operatorname{Ker}\left(1-w_{i_{0}}^{\dagger}\right)$ of

$$
\begin{aligned}
& \mathbf{c}\left(P_{k_{0}}\left|A_{k_{0}}: P_{i_{0}}\right| A_{i_{0}}: w_{k_{0} i_{0}}^{\dagger}: \Lambda_{i_{0}}\right) \\
& \quad \bullet \amalg_{\boldsymbol{\theta}_{i_{0}}}^{w_{i_{0}}^{\dagger}}(\Lambda) \bullet \mathbf{c}\left(P_{i_{0}}\left|A_{i_{0}}: P_{k_{0}}\right| A_{k_{0}}: w_{k_{0} i_{0}}^{-\dagger}: \Lambda_{k_{0}}+w_{k_{0} i_{0}}^{\dagger} \Lambda\right)
\end{aligned}
$$


or still $\mathrm{\amalg}_{\boldsymbol{\theta}_{k_{0}}}^{w_{k_{0}}^{\dagger}}(0)$, where $\boldsymbol{\theta}_{k_{0}}$ is the Detroit family given by

$$
\begin{aligned}
{\left[\theta_{k_{0}}\right]_{\mathscr{E}^{\dagger}}(\Lambda)=} & \mathbf{c}\left(P_{k_{0}}\left|A_{k_{0}}: P_{i_{0}}\right| A_{i_{0}}: w_{k_{0} i_{0}}^{\dagger}: \Lambda_{i_{0}}\right) \\
& \bullet \mathbf{c}\left(P_{i_{0}}\left|A_{i_{0}}: P_{j_{0}}\right| A_{j_{0}}: w_{i_{0}}^{-\dagger} w_{j_{0} i_{0}}^{-\dagger}: w_{j_{0} i_{0}}^{\dagger} w_{i_{0}}^{\dagger} \Lambda_{i_{0}}\right) \\
& \mathbf{e}\left(\mathscr{C}_{j_{0}}: \mathbf{H}: w_{j_{0} i_{0}}^{\dagger} \Lambda\right) \\
& \mathbf{c}\left(P_{j_{0}}\left|A_{j_{0}}: P_{i_{0}}\right| A_{i_{0}}: w_{j_{0} i_{0}}^{\dagger}: \Lambda+w_{i_{0}}^{\dagger} \Lambda_{i_{0}}\right) \\
& \bullet \mathbf{c}\left(P_{i_{0}}\left|A_{i_{0}}: P_{k_{0}}\right| A_{k_{0}}: w_{k_{0} i_{0}}^{-\dagger}: \Lambda_{k_{0}}+w_{k_{0} i_{0}}^{\dagger} \Lambda\right)
\end{aligned}
$$

if $\mathscr{C}^{\dagger} \leftrightarrow\left(j_{0}, w_{j_{0} i_{0}}^{\dagger}\right)$. Note that we have used the well-known formula for the adjoint of a c-function (cf. TES, p. 318), as well as the fact that $\Lambda_{i_{0}}$ is pure imaginary (to get rid of the conjugate). Now $w_{i_{0}}^{\dagger} \Lambda_{i_{0}}=\Lambda_{i_{0}}$, so, from the functional equations,

$$
\begin{aligned}
& \mathbf{c}\left(P_{k_{0}}\left|A_{k_{0}}: P_{i_{0}}\right| A_{i_{0}}: w_{k_{0} i_{0}}^{\dagger}: \Lambda_{i_{0}}\right) \\
& \bullet \mathbf{c}\left(P_{i_{0}}\left|A_{i_{0}}: P_{j_{0}}\right| A_{j_{0}}: w_{i_{0}}^{-\dagger} w_{j_{0} i_{0}}^{-\dagger}: w_{j_{0} i_{0}}^{\dagger} w_{i_{0}}^{\dagger} \Lambda_{i_{0}}\right) \\
&= \mathbf{c}\left(P_{k_{0}}\left|A_{k_{0}}: P_{j_{0}}\right| A_{j_{0}}: w_{k_{0} i_{0}}^{\dagger} w_{i_{0}}^{-\dagger} w_{j_{0} i_{0}}^{-\dagger}: w_{j_{0} i_{0}}^{\dagger} \Lambda_{i_{0}}\right) \\
&= \mathbf{c}\left(P_{k_{0}}\left|A_{k_{0}}: P_{j_{0}}\right| A_{j_{0}}: w_{k_{0}}^{-\dagger} w_{k_{0} i_{0}}^{\dagger} w_{j_{0} i_{0}}^{-\dagger}: w_{j_{0} i_{0}}^{\dagger} \Lambda_{i_{0}}\right) \\
&= \mathbf{c}\left(P_{k_{0}}\left|A_{k_{0}}: P_{k_{0}}\right| A_{k_{0}}: w_{k_{0}}^{\dagger}: \Lambda_{k_{0}}\right) \\
& \bullet \mathbf{c}\left(P_{k_{0}}\left|A_{k_{0}}: P_{j_{0}}\right| A_{j_{0}}: w_{k_{0} i_{0}}^{\dagger} w_{j_{0} i_{0}}^{-\dagger}: w_{j_{0} i_{0}}^{\dagger} \Lambda_{i_{0}}\right) \\
&= \mathbf{c}\left(P_{k_{0}}\left|A_{k_{0}}: P_{k_{0}}\right| A_{k_{0}}: w_{k_{0}}^{-\dagger}: 0\right) \\
& \bullet \mathbf{c}\left(P_{k_{0}}\left|A_{k_{0}}: P_{j_{0}}\right| A_{j_{0}}: w_{k_{0} i_{0}}^{\dagger} w_{j_{0} i_{0}}^{-\dagger}: w_{j_{0} i_{0}}^{\dagger} \Lambda_{i_{0}}\right),
\end{aligned}
$$

the rationale behind the last step being that

$$
\mathbf{c}\left(P_{k_{0}}\left|A_{k_{0}}: P_{k_{0}}\right| A_{k_{0}}: w_{k_{0}}^{-\dagger}: 0\right)
$$

depends only on the component of $\Lambda_{k_{0}}$ in $\operatorname{Im}\left(1-w_{k_{0}}^{-\dagger}\right)$. Similarly,

$$
\begin{aligned}
\mathbf{c}\left(P_{j_{0}}\left|A_{j_{0}}: P_{i_{0}}\right| A_{i_{0}}: w_{j_{0} i_{0}}^{\dagger}: \Lambda+w_{i_{0}}^{\dagger} \Lambda_{i_{0}}\right) & \\
& \bullet \mathbf{c}\left(P_{i_{0}}\left|A_{i_{0}}: P_{k_{0}}\right| A_{k_{0}}: w_{k_{0} i_{0}}^{-\dagger}: \Lambda_{k_{0}}^{\dagger}+w_{k_{0} i_{0}}^{\dagger} \Lambda\right) \\
= & \mathbf{c}\left(P_{j_{0}}\left|A_{j_{0}}: P_{k_{0}}\right| A_{k_{0}}: w_{j_{0} i_{0}}^{\dagger} w_{k_{0} i_{0}}^{-\dagger}: \Lambda_{k_{0}}+w_{k_{0} i_{0}}^{\dagger} \Lambda\right) .
\end{aligned}
$$

All this suggests that a slight change in notations is appropriate, namely let still $\boldsymbol{\theta}_{k_{0}}$ be the Detroit family given by

$$
\begin{aligned}
{\left[\theta_{k_{0}}\right]_{\mathscr{C}^{\dagger}}(\Lambda)=} & \mathbf{c}\left(P_{k_{0}}\left|A_{k_{0}}: P_{j_{0}}\right| A_{j_{0}}: w_{j_{0} k_{0}}^{-\dagger}: w_{j_{0} k_{0}}^{\dagger} \Lambda_{k_{0}}\right) \\
& \times \mathbf{e}\left(\mathscr{C}_{j_{0}}: \mathbf{H}: w_{j_{0} k_{0}}^{\dagger} \Lambda\right) \\
& \mathbf{c}\left(P_{j_{0}}\left|A_{j_{0}}: P_{k_{0}}\right| A_{k_{0}}: w_{j_{0} k_{0}}^{\dagger}: \Lambda_{k_{0}}+\Lambda\right)
\end{aligned}
$$


if $\mathscr{C}^{\dagger} \leftrightarrow\left(j_{0}, w_{j_{0} i_{0}}^{\dagger}\right)$ and $w_{j_{0} k_{0}}^{\dagger} \equiv w_{j_{0} i_{0}}^{\dagger} w_{k_{0} i_{0}}^{-\dagger}$. Thus, over at $\mathscr{C}_{k_{0}}$, we have

$$
\begin{aligned}
\operatorname{tr}_{\mathscr{B}_{i}}\left(\mathbf{W}_{\boldsymbol{\theta}_{k_{0}}}^{w_{k_{0}}^{\dagger}}(0)\right. & \bullet \mathbf{c}\left(P_{k_{0}}\left|A_{k_{0}}: P_{k_{0}}\right| A_{k_{0}}: w_{k_{0}}^{-\dagger}: 0\right) \\
& \left.\bullet \operatorname{Ind}_{\mathscr{C}_{k_{0}}}^{G}\left(\left(\mathscr{O}_{k_{0}}, \Lambda_{k_{0}}\right)\right)(\alpha)\right) .
\end{aligned}
$$

If $\left\{w_{k_{0}}^{\dagger}\right\}$ is a set of representatives for the association classes of $w_{i_{0}}^{\dagger}$ (cf. [11, $\S 10])$, then all $w_{i_{0}}^{\dagger}$ associate to a fixed $w_{k_{0}}^{\dagger}$ are going to lead to the same result; there are $*\left(\mathscr{C}_{i_{0}}^{\dagger}\right) / \#\left(\operatorname{Cen}\left(w_{k_{0}}^{\dagger}\right)\right)$ such terms. Replacing $k_{0}$ by $i_{0}$, the focus of our investigation becomes

$$
\begin{aligned}
& \sum_{\mathscr{E}_{i}, \mathscr{V}_{i_{0}}} \sum_{\mathscr{O}_{i_{0}}}\left\langle\mathbf{p}\left(\Gamma: \mathscr{C}_{i}: \mathbf{H}-\mathbf{H}_{\mathrm{O}}\right),\right. \\
& \sum_{\left\{w_{i_{0}}^{\dagger}\right\}}\left(-\frac{1}{2 \pi}\right)^{l_{0}-\operatorname{rank}\left(1-w_{i_{0}}^{\dagger}\right)} \\
& \text { - } \frac{1}{\left|\operatorname{det}\left(\left(1-w_{i_{0}}^{\dagger}\right) \mid \operatorname{Im}\left(1-w_{i_{0}}^{\dagger}\right)\right)\right|} \cdot \frac{1}{\#\left(\operatorname{Cen}\left(w_{i_{0}}^{\dagger}\right)\right)} \\
& \times \int_{\sqrt{-1} \operatorname{Ker}\left(1-w_{i_{0}}^{\dagger}\right)} \operatorname{tr}_{\mathscr{C}_{i}}\left(\amalg_{\boldsymbol{\theta}_{i_{0}}}^{w_{i_{0}}^{\dagger}}(0)\right. \\
& \left.\left.\times \mathbf{c}\left(P_{i_{0}}\left|A_{i_{0}}: P_{i_{0}}\right| A_{i_{0}}: w_{i_{0}}^{-\dagger}: 0\right) \bullet \mathbf{I n d}_{\mathscr{C}_{i_{0}}}^{G}\left(\left(\mathscr{O}_{i_{0}}, \Lambda_{i_{0}}\right)\right)(\alpha)\right)\right\rangle\left|d \Lambda_{i_{0}}\right| .
\end{aligned}
$$

There is certainly no loss of generality in taking the $w_{i_{0}}^{\dagger}$ simple in the sense that $\operatorname{Ker}\left(1-w_{i_{0}}^{\dagger}\right)$ is a split component, say $\mathfrak{a}_{i_{0}}^{\prime \prime}\left(w_{i_{0}}^{\dagger}\right)$ :

$$
\mathfrak{a}_{i_{0}} \supset \mathfrak{a}_{i_{0}}^{\prime \prime}\left(w_{i_{0}}^{\dagger}\right) \supset \mathfrak{a}_{i}
$$

Moreover, if $w_{i_{0}}^{\dagger}$ runs through a set of representatives, then so does $w_{i_{0}}^{-\dagger}$. Since

$$
\operatorname{Ker}\left(1-w_{i_{0}}^{\dagger}\right)=\operatorname{Ker}\left(1-w_{i_{0}}^{-\dagger}\right)
$$

everything stays the same except for the replacement of

$$
\mathbf{c}\left(P_{i_{0}}\left|A_{i_{0}}: P_{i_{0}}\right| A_{i_{0}}: w_{i_{0}}^{-\dagger}: 0\right)
$$

by

$$
\mathbf{c}\left(P_{i_{0}}\left|A_{i_{0}}: P_{i_{0}}\right| A_{i_{0}}: w_{i_{0}}^{\dagger}: 0\right) \text {. }
$$

Remark. To motivate the use of the term simple, we remark that if $w$ is a reflection in a root $\lambda$, then $w$ is simple iff $\lambda$ is simple, i.e., the two possible meanings of the adjective "simple" in the phrase "simple reflection" are the same. 
To make further progress, it will be necessary to express $\boldsymbol{\theta}_{i_{0}}$ as a product of two Detroit families. For this purpose, fix an $\mathbf{H}_{\mathrm{OO}} \in \mathfrak{a}$ and note that

$$
\begin{aligned}
& \mathbf{c}\left(P_{j_{0}}\left|A_{j_{0}}: P_{i_{0}}\right| A_{i_{0}}: w_{j_{0} i_{0}}^{\dagger}: \Lambda_{i_{0}}\right) \\
& \quad \bullet \mathbf{c}\left(P_{i_{0}}\left|A_{i_{0}}: P_{j_{0}}\right| A_{j_{0}}: w_{j_{0} i_{0}}^{-\dagger}: w_{j_{0} i_{0}}^{\dagger} \Lambda_{i_{0}}\right)=1 .
\end{aligned}
$$

Then

$$
\boldsymbol{\theta}_{i_{0}}=\phi_{i_{0}} \bullet \boldsymbol{\psi}_{i_{0}}
$$

where

$$
\begin{aligned}
{\left[\phi_{i_{0}}\right]_{\mathscr{C}^{\dagger}}(\Lambda)=} & \mathbf{c}\left(P_{i_{0}}\left|A_{i_{0}}: P_{j_{0}}\right| A_{j_{0}}: w_{j_{0} i_{0}}^{-\dagger}: w_{j_{0} i_{0}}^{\dagger} \Lambda_{i_{0}}\right) \\
& \times \mathbf{e}\left(\mathscr{C}_{j_{0}}: \mathbf{H}-\mathbf{H}_{\mathrm{OO}}: w_{j_{0} i_{0}}^{\dagger} \Lambda\right) \\
& \mathbf{c}\left(P_{j_{0}}\left|A_{j_{0}}: P_{i_{0}}\right| A_{i_{0}}: w_{j_{0} i_{0}}^{\dagger}: \Lambda_{i_{0}}\right)
\end{aligned}
$$

and

$$
\begin{aligned}
{\left[\psi_{i_{0}}\right]_{\mathscr{C}^{\dagger}}(\Lambda)=} & \mathbf{c}\left(P_{i_{0}}\left|A_{i_{0}}: P_{j_{0}}\right| A_{j_{0}}: w_{j_{0} i_{0}}^{-\dagger}: w_{j_{0} i_{0}}^{\dagger} \Lambda_{i_{0}}\right) \\
& \times \mathbf{e}\left(\mathscr{C}_{j_{0}}: \mathbf{H}_{\mathrm{OO}}: w_{j_{0} i_{0}}^{\dagger} \Lambda\right) \\
& \mathbf{c}\left(P_{j_{0}}\left|A_{j_{0}}: P_{i_{0}}\right| A_{i_{0}}: w_{j_{0} i_{0}}^{\dagger}: \Lambda_{i_{0}}+\Lambda\right)
\end{aligned}
$$

if $\mathscr{C}^{\dagger} \leftrightarrow\left(j_{0}, w_{j_{0} i_{0}}^{\dagger}\right)$.

This means that the evaluation of $\amalg_{\theta_{i_{0}}}^{w_{i_{0}}^{\dagger}}(0)$ can be handled by an appeal to Theorem 4.4 of [11]. Here,

$$
\left(\mathscr{C}_{W}, \mathscr{C}(W)\right) \leftrightarrow \mathscr{C}^{\dagger} \leftrightarrow\left(j_{0}, w_{j_{0} i_{0}}^{\dagger}\right),
$$

with

$$
\mathfrak{a}_{i_{0}} \supset \mathfrak{a}_{i_{0}}^{\prime \prime}\left(w_{i_{0}}^{\dagger}\right) \supset W^{\perp} \oplus \mathfrak{a}_{i} \supset \mathfrak{a}_{i} .
$$

We are therefore led to

$$
\begin{aligned}
& \sum_{\mathscr{E}_{i}, \mathscr{C}_{i_{0}}} \sum_{\mathscr{O}_{i_{0}}}\left\langle\mathbf{p}\left(\Gamma: \mathscr{C}_{i}: \mathbf{H}-\mathbf{H}_{\mathrm{O}}\right),\right. \\
& \sum_{\left\{w_{i_{0}}^{\dagger}\right\}}\left(-\frac{1}{2 \pi}\right)^{l_{0}-\operatorname{rank}\left(1-w_{i_{0}}^{\dagger}\right)} \\
& \text { - } \frac{1}{\left|\operatorname{det}\left(\left(1-w_{i_{0}}^{\dagger}\right) \mid \operatorname{Im}\left(1-w_{i_{0}}^{\dagger}\right)\right)\right|} \cdot \frac{1}{\#\left(\operatorname{Cen}\left(w_{i_{0}}^{\dagger}\right)\right)} \\
& \left.\times \sum_{W, \mathscr{C}_{W}}(-1)^{\operatorname{dim}\left(W^{\perp}\right)} \int_{\sqrt{-1} \mathfrak{a}_{i_{0}}^{\prime \prime}\left(w_{i_{0}}^{\dagger}\right)} \operatorname{tr}_{\mathscr{C}_{i}}(?)\right\rangle\left|d \Lambda_{i_{0}}^{\prime \prime}\right| \text {, }
\end{aligned}
$$


the ? inside the trace being the product of

$$
\begin{aligned}
& \mathbf{c}\left(P_{i_{0}}\left|A_{i_{0}}: P_{j_{0}}\right| A_{j_{0}}: w_{j_{0} i_{0}}^{-\dagger}: w_{j_{0} i_{0}}^{\dagger} \Lambda_{i_{0}}\right) \\
& \quad \bullet \mathbf{p}\left(\Gamma: \mathscr{C}_{j_{0}}: \mathbf{H}-\mathbf{H}_{\mathrm{OO}}\right) \bullet \mathbf{c}\left(P_{j_{0}}\left|A_{j_{0}}: P_{i_{0}}\right| A_{i_{0}}: w_{j_{0} i_{0}}^{\dagger}: \Lambda_{i_{0}}\right)
\end{aligned}
$$

and $\mathrm{W}_{\boldsymbol{\psi}_{i_{0}}\left(\mathscr{E}_{W}\right)}^{w_{i_{0}}^{\dagger}}(0)$ and

$$
\mathbf{c}\left(P_{i_{0}}\left|A_{i_{0}}: P_{i_{0}}\right| A_{i_{0}}: w_{i_{0}}^{\dagger}: 0\right) \bullet \operatorname{Ind}_{\mathscr{C}_{i_{0}}}^{G}\left(\left(\mathscr{O}_{i_{0}}, \Lambda_{i_{0}}\right)\right)(\alpha) .
$$

The appearance of the Arthur polynomial at the ${ }^{\prime} \mathscr{C}_{j_{0}}$-level comes about, of course, from differentiating the exponential.

To simplify the integrand, start by putting it in the form

$$
\left\langle\mathbf{p}\left(\Gamma:{ }^{\prime} \mathscr{C}_{j_{0}}: \mathbf{H}-\mathbf{H}_{\mathrm{OO}}\right), \mathbf{t r}_{\mathscr{C}_{j_{0}}}(?)\right\rangle_{\mathscr{\mathscr { C }}_{i}},
$$

where this time? is the product of

$$
\mathbf{c}\left(P_{j_{0}}\left|A_{j_{0}}: P_{i_{0}}\right| A_{i_{0}}: w_{j_{0} i_{0}}^{\dagger}: \Lambda_{i_{0}}\right) \bullet \amalg_{\psi_{i_{0}}\left(\mathscr{C}_{W}\right)}^{w_{i}^{\dagger}}(0)
$$

and

or still

$$
\left\{\begin{array}{l}
\mathbf{c}\left(P_{i_{0}}\left|A_{i_{0}}: P_{i_{0}}\right| A_{i_{0}}: w_{i_{0}}^{\dagger}: 0\right) \\
\operatorname{Ind}_{\mathscr{G}_{i_{0}}}^{G}\left(\left(\mathscr{O}_{i_{0}}, \Lambda_{i_{0}}\right)\right)(\alpha) \\
\mathbf{c}\left(P_{i_{0}}\left|A_{i_{0}}: P_{j_{0}}\right| A_{j_{0}}: w_{j_{0} i_{0}}^{-\dagger}: w_{j_{0} i_{0}}^{\dagger} \Lambda_{i_{0}}\right)
\end{array}\right.
$$

$$
\left\{\begin{array}{l}
\mathbf{c}\left(P_{i_{0}}\left|A_{i_{0}}: P_{j_{0}}\right| A_{j_{0}}: w_{j_{0} i_{0}}^{-\dagger}: \Lambda_{j_{0}}\right) \\
\mathbf{c}\left(P_{j_{0}}\left|A_{j_{0}}: P_{j_{0}}\right| A_{j_{0}}: w_{j_{0}}^{\dagger}: 0\right) \\
\operatorname{Ind}_{\mathscr{C}_{j_{0}}}^{G}\left(\left(\mathscr{O}_{j_{0}}, \Lambda_{j_{0}}\right)\right)(\alpha)
\end{array}\right.
$$

provided we set $\Lambda_{j_{0}}=w_{j_{0} i_{0}}^{\dagger} \Lambda_{i_{0}}$ and $w_{j_{0}}^{\dagger}=w_{j_{0} i_{0}}^{\dagger} w_{i_{0}}^{\dagger} w_{j_{0} i_{0}}^{-\dagger}$. Using now the device employed earlier, pass to the Detroit family at the base point determined by $\left(j_{0}, w_{j_{0} i_{0}}^{\dagger}\right)$ to conclude that

$$
\mathbf{c}\left(P_{j_{0}}\left|A_{j_{0}}: P_{i_{0}}\right| A_{i_{0}}: w_{j_{0} i_{0}}^{\dagger}: \Lambda_{i_{0}}\right) \bullet \amalg_{\psi_{i_{0}}\left(\mathscr{C}_{W}\right)}^{w_{i^{\prime}}^{\dagger}}(0) \bullet \mathbf{c}\left(P_{i_{0}}\left|A_{i_{0}}: P_{j_{0}}\right| A_{j_{0}}: w_{j_{0} i_{0}}^{-\dagger}: \Lambda_{j_{0}}\right)
$$

is equal to $\amalg_{\psi_{j_{0}}\left(\mathscr{E}_{W}\right)}^{w_{j^{\prime}}^{\dagger}}(0)$, the second $W$ differing from (but corresponding to) the first $W$.

Making these changes, the integral over $\sqrt{-1} \mathfrak{a}_{i_{0}}^{\prime \prime}\left(w_{i_{0}}^{\dagger}\right)$ becomes the integral over $\sqrt{-1} \mathfrak{a}_{j_{0}}^{\prime \prime}\left(w_{j_{0}}^{\dagger}\right)$ of

$$
\begin{aligned}
& \left\langle\mathbf{p}\left(\Gamma:{ }^{\prime} \mathscr{C}_{j_{0}}: \mathbf{H}-\mathbf{H}_{\mathrm{OO}}\right),\right. \\
& \left.\quad \operatorname{tr}_{\mathscr{C}_{j_{0}}}\left(\boldsymbol{W}_{\psi_{j_{0}}\left(\mathscr{C}_{W}\right)}^{w_{\dagger}^{\dagger}}(0) \bullet \mathbf{c}\left(P_{j_{0}}\left|A_{j_{0}}: P_{j_{0}}\right| A_{j_{0}}: w_{j_{0}}^{\dagger}: 0\right) \bullet \operatorname{Ind}_{\mathscr{C}_{j_{0}}}^{G}\left(\left(\mathscr{O}_{j_{0}}, \Lambda_{j_{0}}\right)\right)(\alpha)\right)\right\rangle_{\mathscr{C}_{i}} .
\end{aligned}
$$


To collapse the sum over $\mathscr{C}_{i}$, we shall have to address the combinatorics of the situation, since, after all, some choices have been made and our terms are independent of them. In a word: We must sum over all possible choices and measure the overlap.

To initiate the process, write

$$
\begin{aligned}
\sum_{\mathscr{E}_{i}, \mathscr{C}_{i_{0}} \mathscr{O}_{i_{0}}}\left\langle\mathbf{p}\left(\Gamma: \mathscr{C}_{i}: \mathbf{H}-\mathbf{H}_{\mathrm{O}}\right), \sum_{\left\{w_{i_{0}}^{\dagger}\right\}}\left(-\frac{1}{2 \pi}\right)^{l_{0}-\operatorname{rank}\left(1-w_{i_{0}}^{\dagger}\right)}\right. \\
\quad \cdot \frac{1}{\left|\operatorname{det}\left(\left(1-w_{i_{0}}^{\dagger}\right) \mid \operatorname{Im}\left(1-w_{i_{0}}^{\dagger}\right)\right)\right|} \cdot \frac{1}{\#\left(\operatorname{Cen}\left(w_{i_{0}}^{\dagger}\right)\right)} \\
\quad \times \sum_{W, \mathscr{C}_{W}}(-1)^{\operatorname{dim}\left(W^{\perp}\right)} \\
\left.\quad \times \int_{\sqrt{-1} \mathfrak{a}_{j_{0}}^{\prime \prime}\left(w_{j_{0}}^{\dagger}\right)}\left\langle\mathbf{p}\left(\Gamma:^{\prime} \mathscr{C}_{j_{0}}: \mathbf{H}-\mathbf{H}_{\mathrm{OO}}\right), \mathbf{t r}_{\mathscr{C}_{j_{0}}}(?)\right\rangle_{\mathscr{C}_{i}}\right\rangle\left|d \Lambda_{j_{0}}^{\prime \prime}\right|
\end{aligned}
$$

as

$$
\begin{aligned}
& \sum_{\mathscr{C}_{i}, \mathscr{C}_{i_{0}}} \sum_{\mathscr{O}_{i_{0}}} \sum_{w_{i_{0}}^{\dagger}} \sum_{W, \mathscr{C}_{W}} \frac{(-1)^{\operatorname{dim}\left(\mathfrak{a}_{i_{0}}\right)-\operatorname{dim}\left(\mathfrak{a}_{i}\right)-\operatorname{dim}\left(W^{\perp}\right)-\operatorname{rank}\left(1-w_{i_{0}}^{\dagger}\right)}}{(2 \pi)^{l_{0}-\operatorname{rank}\left(1-w_{i_{0}}^{\dagger}\right)}} \\
& \times \frac{1}{\left|\operatorname{det}\left(\left(1-w_{i_{0}}^{\dagger}\right) \mid \operatorname{Im}\left(1-w_{i_{0}}^{\dagger}\right)\right)\right|} C_{i, i_{0}}\left(w_{i_{0}}^{\dagger}\right) \\
& \times \int_{\sqrt{-1} \mathfrak{a}_{j_{0}}^{\prime \prime}\left(w_{j_{0}}^{\dagger}\right)}\left\langle\mathbf{p}\left(\Gamma: \mathscr{C}_{i}: \mathbf{H}_{\mathrm{O}}-\mathbf{H}\right),\right. \\
&\left.\left\langle\mathbf{p}\left(\Gamma::^{\prime} \mathscr{C}_{j_{0}}: \mathbf{H}-\mathbf{H}_{\mathrm{OO}}\right), \operatorname{tr}_{\mathscr{C}_{j_{0}}}(?)\right\rangle_{\mathscr{C}_{i}}\right\rangle\left|d \Lambda_{j_{0}}^{\prime \prime}\right|,
\end{aligned}
$$

where the sum $\sum_{w_{i_{0}}^{\dagger}}$ extends over all simple $w_{i_{0}}^{\dagger}$. The sign change follows from the homogeneity of the Arthur polynomial:

$$
\mathbf{p}\left(\Gamma: \mathscr{C}_{i}: \mathbf{H}-\mathbf{H}_{\mathrm{O}}\right)=(-1)^{l_{i}} \bullet \mathbf{p}\left(\Gamma: \mathscr{C}_{i}: \mathbf{H}_{\mathrm{O}}-\mathbf{H}\right) .
$$

As for the constant $C_{i, i_{0}}\left(w_{i_{0}}^{\dagger}\right)$, we have

Lemma 4.1. Under the preceding hypotheses,

$$
C_{i, i_{0}}\left(w_{i_{0}}^{\dagger}\right)=\frac{1}{*\left(\mathscr{C}_{i_{0}}\left(w_{i_{0}}^{\dagger}\right)\right) \bullet *\left(\mathscr{C}_{i_{0}}^{\dagger^{\prime \prime}}\right)} .
$$

Proof. To reduce the notational complexity of the calculation, we shall assume that $\mathscr{C}_{i}=\{G\}$. Obviously, $C_{i, i_{0}}\left(w_{i_{0}}\right)$ is equal to $1 / \#\left(\operatorname{Cen}\left(w_{i_{0}}\right)\right)$ divided by the number of simple $w_{j_{0}}$ in the association class, itself the cardinality of $\left\{w_{j_{0} i_{0}}\right.$ : $w_{j_{0} i_{0}} w_{i_{0}} w_{j_{0} i_{0}}^{-1}$ simple $\}$ divided by $\#\left(\operatorname{Cen}\left(w_{i_{0}}\right)\right)$. So,

$$
C_{i, i_{0}}\left(w_{i_{0}}\right)=\frac{1}{\#\left(\left\{w_{j_{0} i_{0}}: w_{j_{0} i_{0}} w_{i_{0}} w_{j_{0} i_{0}}^{-1} \text { simple }\right\}\right)} .
$$


By definition, $w_{j_{0}}=w_{j_{0} i_{0}} w_{i_{0}} w_{j_{0} i_{0}}^{-1}$ is simple iff $w_{j_{0} i_{0}} \bullet \operatorname{Ker}\left(1-w_{i_{0}}\right)$ is a split component. The corresponding parabolics $P_{j_{0}}^{\prime \prime}\left(w_{j_{0}}\right)$ and $P_{i_{0}}^{\prime \prime}\left(w_{i_{0}}\right)$ are then associate with

$$
w_{j_{0} i_{0}} \in W\left(A_{j_{0}}^{\prime \prime}\left(w_{j_{0}}\right), A_{i_{0}}^{\prime \prime}\left(w_{i_{0}}\right)\right) \text {. }
$$

On the other hand, any associate $P^{\prime \prime}$ of $P_{i_{0}}^{\prime \prime}\left(w_{i_{0}}\right)$ must dominate a $P_{j_{0}}$ for which there exists a $w_{j_{0} i_{0}}$ such that

$$
\mathfrak{a}^{\prime \prime}=w_{j_{0} i_{0}}\left(\mathfrak{a}_{i_{0}}^{\prime \prime}\left(w_{i_{0}}\right)\right) \text {. }
$$

Because the totality of all such restrictions is $*\left(" \mathscr{C}_{i_{0}}\left(w_{i_{0}}\right)\right), C_{i, i_{0}}\left(w_{i_{0}}\right)$ is equal to

$$
\frac{1}{*\left(" \mathscr{C}_{i_{0}}\left(w_{i_{0}}\right)\right)} \times \frac{1}{\#\left(\left\{w_{j_{0} i_{0}}: w_{j_{0} i_{0}} \mid \mathfrak{a}_{i_{0}}\left(w_{i_{0}}\right)=w\right\}\right)},
$$

$w$ fixed. Now if $w_{j_{0} i_{0}}\left|\mathfrak{a}_{i_{0}}\left(w_{i_{0}}\right), w_{k_{0} i_{0}}\right| \mathfrak{a}_{i_{0}}\left(w_{i_{0}}\right)=w$, then

$$
w_{k_{0} i_{0}} w_{j_{0} i_{0}}^{-1} \equiv w_{k_{0} j_{0}}^{\dagger^{\prime \prime}} \in W\left(A_{k_{0}}^{\dagger^{\prime \prime}}, A_{j_{0}}^{\dagger^{\prime \prime}}\right),
$$

while, conversely, if $w_{k_{0} j_{0}}^{\dagger^{\prime \prime}} \in W\left(A_{k_{0}}^{\dagger \prime \prime}, A_{j_{0}}^{\dagger^{\prime \prime}}\right)$, then $w_{k_{0} j_{0}}^{\dagger^{\prime \prime}} w_{j_{0} i_{0}}$ and $w_{j_{0} i_{0}}$ have the same restriction to $\mathfrak{a}_{i_{0}}^{\prime \prime}\left(w_{i_{0}}\right)$. Consequently,

$$
\frac{1}{\#\left(\left\{w_{j_{0} i_{0}}: w_{j_{0} i_{0}} \mid \mathfrak{a}_{i_{0}}\left(w_{i_{0}}\right)=w\right\}\right)}=\frac{1}{*\left(\mathscr{C}_{i_{0}}^{\dagger \prime}\right)} .
$$

Hence the lemma.

Continuing the process, let

$$
\mathfrak{a}_{j_{0}}^{\prime}=w_{j_{0} i_{0}}^{\dagger} W^{\perp}+\mathfrak{a}_{i}
$$

and write

$$
\begin{aligned}
& \sum_{\mathscr{C}_{i}, \mathscr{C}_{i_{0}}} \sum_{\mathscr{O}_{i_{0}}} \sum_{w_{i_{0}}^{\dagger}} \sum_{W, \mathscr{C}_{W}} \frac{(-1)^{\operatorname{dim}\left(\mathfrak{a}_{i_{0}}\right)-\operatorname{dim}\left(\mathfrak{a}_{i}\right)-\operatorname{dim}\left(W^{\perp}\right)-\operatorname{rank}\left(1-w_{i_{0}}^{\dagger}\right)}}{(2 \pi)^{l_{0}-\operatorname{rank}\left(1-w_{i_{0}}^{\dagger}\right)}} \\
& \quad \times \frac{1}{\left|\operatorname{det}\left(\left(1-w_{i_{0}}^{\dagger}\right) \mid \operatorname{Im}\left(1-w_{i_{0}}^{\dagger}\right)\right)\right|} \\
& \quad \times \frac{1}{*\left({ }^{\prime \prime} \mathscr{C}_{i_{0}}\left(w_{i_{0}}^{\dagger}\right)\right) \bullet *\left(\mathscr{C}_{i_{0}}^{\dagger \prime \prime}\right)} \\
& \quad \times \int_{\sqrt{-1} a_{j_{0}}^{\prime \prime}\left(w_{j_{0}}^{\dagger}\right)}^{\left\langle\mathbf{p}\left(\Gamma: \mathscr{C}_{i}: \mathbf{H}_{\mathrm{O}}-\mathbf{H}\right),\right.} \\
& \left\langle\mathbf{p}\left(\Gamma: \mathscr{C}_{j_{0}}: \mathbf{H}-\mathbf{H}_{\mathrm{OO}}\right), \mathbf{t r}_{\mathscr{C}_{j_{0}}}(?)\right\rangle_{\mathscr{C}_{i}}\left|d \Lambda_{j_{0}}^{\prime \prime}\right|
\end{aligned}
$$


as

$$
\begin{aligned}
& \sum_{\mathscr{C}_{i}, \mathscr{C}_{j_{0}}} \sum_{\mathscr{O}_{j_{0}}} \sum_{w_{j_{0}}^{\dagger}} \sum_{\mathfrak{a}_{j_{0}}^{\prime}} \frac{(-1)^{\operatorname{dim}\left(\mathfrak{a}_{j_{0}}\right)-\operatorname{dim}\left(\mathfrak{a}_{j_{0}}^{\prime}\right)-\operatorname{rank}\left(1-w_{j_{0}}^{\dagger}\right)}}{(2 \pi)^{l_{0}-\operatorname{rank}\left(1-w_{j_{0}}^{\dagger}\right)}} \\
& \times \frac{1}{\left|\operatorname{det}\left(\left(1-w_{j_{0}}^{\dagger}\right) \mid \operatorname{Im}\left(1-w_{j_{0}}^{\dagger}\right)\right)\right|} C_{i, j_{0}}\left(w_{j_{0}}^{\dagger}\right) \\
& \times \int_{\sqrt{-1} \mathfrak{a}_{j_{0}}^{\prime \prime}\left(w_{j_{0}}^{\dagger}\right)}\left\langle\mathbf{p}\left(\Gamma: \mathscr{C}_{i}: \mathbf{H}_{\mathrm{O}}-\mathbf{H}\right),\right. \\
& \left.\left\langle\mathbf{p}\left(\Gamma:{ }^{\prime} \mathscr{C}_{j_{0}}: \mathbf{H}-\mathbf{H}_{\mathrm{OO}}\right), \mathbf{t r}_{\mathscr{C}_{j_{0}}}(?)\right\rangle_{\mathscr{C}_{i}}\right\rangle\left|d \Lambda_{j_{0}}^{\prime \prime}\right| .
\end{aligned}
$$

To reflect this change in emphasis, call $\amalg_{\psi_{j_{0}}^{\dagger}}^{w_{j_{0}}^{\dagger}}$ the Ш-transform figuring in the integral. As for the constant $C_{i, j_{0}}\left(w_{j_{0}}^{\dagger}\right)$, we have

Lemma 4.2. Under the preceding hypotheses,

$$
C_{i, j_{0}}\left(w_{j_{0}}^{\dagger}\right)=\frac{1}{*\left(\mathscr{C}_{j_{0}}^{\prime}\left(w_{j_{0}}^{\dagger}\right)\right) \bullet *\left(\mathscr{C}_{j_{0}}^{t^{\prime \prime}}\right)} .
$$

Proof. To reduce the notational complexity of the calculation, we shall assume that $\mathscr{C}_{i}=\{G\}$. It is then a question of examining

$$
\sum_{w_{i_{0}}} \sum_{W, \mathscr{C}_{W}} \frac{1}{*\left({ }^{\prime \prime} \mathscr{C}_{i_{0}}\left(w_{i_{0}}\right)\right) \bullet *\left(\mathscr{C}_{i_{0}}^{t^{\prime \prime}}\right)}
$$

or still

$$
\sum_{w_{i_{0}}} \sum_{W, \mathscr{C}_{W}} \sum_{\mathscr{C}(W)} \frac{1}{*\left({ }^{\prime \prime} \mathscr{C}_{i_{0}}\left(w_{i_{0}}\right)\right) \bullet *\left(\mathscr{C}_{i_{0}}^{t^{\prime \prime}}\right) \bullet *(\mathscr{C}(W))}
$$

if $\left(\mathscr{C}_{W}, \mathscr{C}(W)\right) \leftrightarrow \mathscr{C} \leftrightarrow w_{j_{0} i_{0}}$. From the definitions,

$$
*(\mathscr{C}(W))=*\left({ }^{\prime} \mathscr{C}_{j_{0}}^{\prime}\left(w_{j_{0}}\right)\right)
$$

where, needless to say, $w_{j_{0}}=w_{j_{0} i_{0}} w_{i_{0}} w_{j_{0} i_{0}}^{-1}$. To reduce the sum, note that quadruples $\left(w_{i_{0}}, W, \mathscr{C}_{W}, \mathscr{C}(W)\right)$ give rise to pairs $\left(w_{j_{0}}, \mathfrak{a}_{j_{0}}^{\prime}\right)$. The number of quadruples corresponding to a given pair is, as can be checked without difficulty, the cardinality of $\left\{w_{j_{0} i_{0}}: w_{j_{0} i_{0}} w_{i_{0}} w_{j_{0} i_{0}}^{-1}\right.$ simple $\}$ divided by the number of $w_{j_{0} i_{0}}$ attached to a fixed $\left(w_{i_{0}}, W, \mathscr{C}_{W}, \mathscr{C}(W)\right)$. The (proof of) preceding lemma computes the first of these. To get at the second, suppose that we have two such attached to $\left(w_{i_{0}}, W, \mathscr{C}_{W}, \mathscr{C}(W)\right)$. Then the product of the one with the inverse of the other must be an element of $W\left(A_{j_{0}}^{\dagger^{\prime \prime}}\right)$. Moreover, if $w_{j_{0}}^{\dagger^{\prime \prime}} \in W\left(A_{j_{0}}^{\dagger^{\prime \prime}}\right)$, then $w_{j_{0}}^{\dagger^{\prime \prime}} w_{j_{0} i_{0}}$ and $w_{j_{0} i_{0}}$ yield the same $\left(W, \mathscr{C}_{W}, \mathscr{C}(W)\right)$ and, finally, give the $w_{i_{0}}$ iff

$$
w_{j_{0}}^{\dagger^{\prime \prime}} \in \operatorname{Cen}^{\dagger^{\prime \prime}}\left(w_{j_{0}}\right)
$$


the centralizer of $w_{j_{0}}$ being taken in $W\left(A_{j_{0}}^{\dagger^{\prime \prime}}\right)$. Let us now declare two quadruples to be equivalent if they give rise to the same pair $\left(w_{j_{0}}, \mathfrak{a}_{j_{0}}^{\prime}\right)$. Then, from the above,

$$
\begin{gathered}
\sum_{w_{i_{0}}} \sum_{W, \mathscr{C}_{W}} \sum_{\mathscr{C}(W)} \frac{1}{*\left({ }^{\prime \prime} \mathscr{C}_{i_{0}}\left(w_{i_{0}}\right)\right) \bullet *\left(\mathscr{C}_{i_{0}}^{\dagger^{\prime \prime}}\right) \bullet *(\mathscr{C}(W))} \\
=\sum_{\operatorname{REP}} \frac{1}{*\left(\mathscr{C}_{j_{0}}^{\prime}\left(w_{j_{0}}\right)\right) \bullet \#\left(\operatorname{Cen}^{\dagger^{\prime \prime}}\left(w_{j_{0}}\right)\right)},
\end{gathered}
$$

the second sum over a set of representatives for the equivalence classes or still over all pairs $\left(w_{j_{0}}, \mathfrak{a}_{j_{0}}^{\prime}\right)$ so long as we compensate by dividing by the number of $\left(w_{j_{0}}, \mathfrak{a}_{j_{0}}^{\prime}\right)$ giving the equivalence class. But if

then actually

$$
\left(w_{i_{0}}, W, \mathscr{C}_{W}, \mathscr{C}(W)\right) \longrightarrow\left(w_{j_{0}}, \mathfrak{a}_{j_{0}}^{\prime}\right)
$$

and

$$
\mathfrak{a}_{j_{0}}^{\prime \prime}\left(w_{j_{0}}\right)=\mathfrak{a}_{k_{0}}^{\prime \prime}\left(w_{k_{0}}\right)
$$

$$
w_{k_{0} i_{0}} w_{j_{0} i_{0}}^{-1} \in W\left(A_{k_{0}}^{\dagger^{\prime \prime}}, A_{j_{0}}^{\dagger^{\prime \prime}}\right) \text {. }
$$

In addition, $\mathfrak{a}_{j_{0}}^{\prime}=\mathfrak{a}_{k_{0}}^{\prime}$. As the story can be read backwards, the constant of compensation is

Hence the lemma.

$$
\frac{*\left(\mathscr{C}_{j_{0}}^{\dagger^{\prime \prime}}\right)}{\#\left(\operatorname{Cen}^{\dagger^{\prime \prime}}\left(w_{j_{0}}\right)\right)} \text {. }
$$

In particular, then,

$$
C_{i, j_{0}}\left(w_{j_{0}}^{\dagger}\right)=\frac{1}{*\left(\mathscr{C}_{j_{0}}^{\prime}\left(w_{j_{0}}^{\dagger}\right)\right) \bullet *\left(\mathscr{C}_{j_{0}}^{\dagger^{\prime \prime}}\right)}
$$

does not depend on $\mathscr{C}_{i}$. We can therefore write

$$
\begin{aligned}
& \sum_{\mathscr{E}_{i}, \mathscr{C}_{j_{0}}} \sum_{\mathcal{O}_{j_{0}}} \sum_{w_{j_{0}}^{\dagger}} \sum_{\mathfrak{a}_{j_{0}}^{\prime}} \frac{(-1)^{\operatorname{dim}\left(\mathfrak{a}_{j_{0}}\right)-\operatorname{dim}\left(\mathfrak{a}_{j_{0}}^{\prime}\right)-\operatorname{rank}\left(1-w_{j_{0}}^{\dagger}\right)}}{(2 \pi)^{l_{0}-\operatorname{rank}\left(1-w_{j_{0}}^{\dagger}\right)}} \\
& \times \frac{1}{\left|\operatorname{det}\left(\left(1-w_{j_{0}}^{\dagger}\right) \mid \operatorname{Im}\left(1-w_{j_{0}}^{\dagger}\right)\right)\right|} \\
& \times \frac{1}{*\left({ }^{\prime} \mathscr{C}_{j_{0}}^{\prime}\left(w_{j_{0}}^{\dagger}\right)\right) \bullet *\left(\mathscr{C}_{j_{0}}^{\dagger^{\prime \prime}}\right)} \\
& \times \int_{\sqrt{-1} \mathfrak{a}_{j_{0}}^{\prime \prime}\left(w_{j_{0}}^{\dagger}\right)}\left\langle\mathbf{p}\left(\Gamma: \mathscr{C}_{i}: \mathbf{H}_{\mathrm{O}}-\mathbf{H}\right),\right. \\
& \left.\left\langle\mathbf{p}\left(\Gamma:{ }^{\prime} \mathscr{C}_{j_{0}}: \mathbf{H}-\mathbf{H}_{\mathrm{OO}}\right), \mathbf{t r}_{\mathscr{C}_{j_{0}}}(?)\right\rangle_{\mathscr{C}_{i}}\right\rangle\left|d \Lambda_{j_{0}}^{\prime \prime}\right|
\end{aligned}
$$


as

$$
\begin{aligned}
& \sum_{\mathscr{C}_{j_{0}}} \sum_{\mathscr{O}_{j_{0}}} \sum_{w_{j_{0}}} \sum_{\mathfrak{a}_{j_{0}}^{\prime}} \frac{(-1)^{\operatorname{dim}\left(\mathfrak{a}_{j_{0}}\right)-\operatorname{dim}\left(\mathfrak{a}_{j_{0}}^{\prime}\right)-\operatorname{rank}\left(1-w_{j_{0}}\right)}}{(2 \pi)^{l_{0}-\operatorname{rank}\left(1-w_{j_{0}}\right)}} \\
& \quad \times \frac{1}{\left|\operatorname{det}\left(\left(1-w_{j_{0}}\right) \mid \operatorname{Im}\left(1-w_{j_{0}}\right)\right)\right|} \\
& \quad \times \frac{1}{*\left({ }^{\prime} \mathscr{C}_{j_{0}}^{\prime}\left(w_{j_{0}}\right)\right) \bullet *\left(\mathscr{C}_{j_{0}}^{\prime \prime}\right)} \\
& \quad \times \int_{\sqrt{-1} a_{j_{0}}^{\prime \prime}\left(w_{j_{0}}\right)}\left\langle\sum_{\mathscr{C}_{i} \succeq \mathscr{C}_{j_{0}}^{\prime}} \mathbf{p}\left(\Gamma: \mathscr{C}_{i}: \mathbf{H}_{\mathrm{O}}-\mathbf{H}\right),\right. \\
& \left.\left\langle\mathbf{p}\left(\Gamma:{ }^{\prime} \mathscr{C}_{j_{0}}: \mathbf{H}-\mathbf{H}_{\mathrm{OO}}\right), \mathbf{t r}_{\mathscr{C}_{j_{0}}}(?)\right\rangle_{\mathscr{C}_{i}}\right\rangle\left|d \Lambda_{j_{0}}^{\prime \prime}\right| .
\end{aligned}
$$

Here we are working with $w_{j_{0}}$ and not $w_{j_{0}}^{\dagger}$ (cf. Lemma 10.1 of [11]). Owing to Proposition 3.2 of [11],

$$
\begin{aligned}
& \left\langle\sum_{\mathscr{C}_{i} \succeq \mathscr{C}_{j_{0}}^{\prime}} \mathbf{p}\left(\Gamma: \mathscr{C}_{i}: \mathbf{H}_{\mathrm{O}}-\mathbf{H}\right),\left\langle\mathbf{p}\left(\Gamma:{ }^{\prime} \mathscr{C}_{j_{0}}: \mathbf{H}-\mathbf{H}_{\mathrm{OO}}\right), \mathbf{t r}_{\mathscr{C}_{j_{0}}}(?)\right\rangle_{\mathscr{C}_{i}}\right\rangle \\
& \quad=\left\langle\mathbf{p}\left(\Gamma: \mathscr{C}_{j_{0}}^{\prime}: \mathbf{H}_{\mathrm{O}}-\mathbf{H}_{\mathrm{OO}}\right), \mathbf{t r}_{\mathscr{C}_{j_{0}}^{\prime}}(?)\right\rangle .
\end{aligned}
$$

But now $\mathbf{H}$ is gone so the cancellation has been carried off.

One can clean things up a bit. Note that

$$
\left\{\begin{array}{l}
\operatorname{dim}\left(\mathfrak{a}_{j_{0}}\right)-\operatorname{dim}\left(\mathfrak{a}_{j_{0}}^{\prime}\right)-\operatorname{rank}\left(1-w_{j_{0}}^{\dagger}\right)=\operatorname{dim}\left(\mathfrak{a}_{j_{0}}^{\prime}\left(w_{j_{0}}^{\dagger}\right)\right), \\
l_{0}-\operatorname{rank}\left(1-w_{j_{0}}^{\dagger}\right)=\operatorname{dim}\left(\mathfrak{a}_{j_{0}}^{\prime \prime}\left(w_{j_{0}}^{\dagger}\right)\right) .
\end{array}\right.
$$

Replacing $\mathbf{H}_{\mathrm{O}}$ by $\mathbf{H}$ and $\mathbf{H}_{\mathrm{OO}}$ by $\mathbf{H}_{\mathrm{O}}$, substituting $\mathfrak{a}_{i}$ for $\mathfrak{a}_{j_{0}}^{\prime}$ and $\mathfrak{a}_{j_{0}}^{\prime}\left(w_{j_{0}}^{\dagger}\right)$ for $\mathfrak{a}_{j_{0}}^{\prime \prime}\left(w_{j_{0}}^{\dagger}\right)$, and changing $j_{0}$ back to $i_{0}$, we end up with

$$
\begin{aligned}
\sum_{\mathscr{C}_{i}, \mathscr{C}_{i_{0}}} \sum_{\mathscr{O}_{i_{0}}} \sum_{w_{i_{0}}^{\dagger}} \frac{(-1)^{\operatorname{dim}\left(\mathfrak{a}_{i_{0}}\left(w_{i_{0}}^{\dagger}\right)\right)}}{(2 \pi)^{\operatorname{dim}\left(\mathfrak{a}_{i_{0}}^{\prime}\left(w_{i_{0}}^{\dagger}\right)\right)}} \\
\times \frac{1}{\left|\operatorname{det}\left(\left(1-w_{i_{0}}^{\dagger}\right) \mid \operatorname{Im}\left(1-w_{i_{0}}^{\dagger}\right)\right)\right|} \times \frac{1}{*\left(\mathscr{C}_{i_{0}}\left(w_{i_{0}}^{\dagger}\right)\right) \bullet *\left(\mathscr{C}_{i_{0}}^{\dagger^{\prime}}\right)} \\
\times \int_{\sqrt{-1} \mathfrak{a}_{i_{0}}^{\prime}\left(w_{i_{0}}^{\dagger}\right)}\left\langle\mathbf{p}\left(\Gamma: \mathscr{C}_{i}: \mathbf{H}-\mathbf{H}_{\mathrm{O}}\right), \mathbf{t r}_{\mathscr{C}_{i}}(?)\right\rangle\left|d \Lambda_{i_{0}}^{\prime}\right|
\end{aligned}
$$

To complete the discussion, we have only to explicate $\operatorname{tr}_{\mathscr{C}_{i}}($ ?). But, in view 
of the definitions, $\mathrm{II}_{\boldsymbol{\psi}_{i_{0}}^{\dagger}}^{w_{i}^{\dagger}}(0)$ is equal to

$$
\begin{aligned}
& \sum_{j_{0},{ }^{\prime} w_{j_{0} i_{0}}} D_{*}^{w_{j_{0}}^{\dagger}}\left[\mathbf{c}\left(P_{i_{0}}\left|A_{i_{0}}: P_{j_{0}}\right| A_{j_{0}}:{ }^{\prime} w_{j_{0} i_{0}}^{-1}: ?\right) \bullet \mathbf{e}\left(\mathscr{C}_{j_{0}}: \mathbf{H}_{\mathrm{O}}: ?\right)\right]{ }_{{ }_{j_{0} i_{0}}} \Lambda_{i_{0}} \\
& \times \mathbf{c}\left(P_{j_{0}}\left|A_{j_{0}}: P_{i_{0}}\right| A_{i_{0}}:{ }^{\prime} w_{j_{0} i_{0}}: \Lambda_{i_{0}}\right) .
\end{aligned}
$$

Here, in the notation of $[11, \S 5]$, the sum $\sum_{j_{0},{ }^{\prime} w_{j_{0} i_{0}}}$ extends over those $j_{0}$ and

$$
\text { ' } w_{j_{0} i_{0}} \in W\left(A_{j_{0}}^{\dagger}, A_{i_{0}}^{\dagger}\right) \text {, }
$$

satisfying the condition ' $w_{j_{0} i_{0}}\left(\Sigma_{P_{i_{0}}^{\dagger^{\prime}}}^{0}\right) \subset \Sigma_{P_{j_{0}}^{\dagger}}^{0}$, while $D_{*}^{w_{j_{0}}^{\dagger}}$ is shorthand for $D_{*}^{\left.\Sigma_{P\left(w_{j}\right)}^{\dagger}\right)}$. Finally, one must multiply up by

$$
\mathbf{c}\left(P_{i_{0}}\left|A_{i_{0}}: P_{i_{0}}\right| A_{i_{0}}: w_{i_{0}}^{\dagger}: 0\right) \bullet \operatorname{Ind}_{\mathscr{C}_{i_{0}}}^{G}\left(\left(\mathscr{O}_{i_{0}}, \Lambda_{i_{0}}\right)\right)(\alpha)
$$

and take the trace.

Reinterpreting $\mathbf{F n c}\left(\mathbf{H}: \mathbf{H}_{\mathrm{O}}: \alpha: \Gamma\right)$, an unambiguous symbol in light of the notational revisions, our main conclusion is then:

Theorem 4.3. $\operatorname{Fnc}\left(\mathbf{H}: \mathbf{H}_{\mathrm{O}}: \alpha: \Gamma\right)$ is equal to

$$
\begin{aligned}
& \sum_{\mathscr{E}_{i}, \mathscr{C}_{i_{0}}} \sum_{\mathscr{O}_{i_{0}}} \sum_{w_{i_{0}}^{\dagger}} \frac{(-1)^{\operatorname{dim}\left({ }^{\prime} a_{i_{0}}\left(w_{i_{0}}^{\dagger}\right)\right)}}{(2 \pi)^{\operatorname{dim}\left(a_{i_{0}}^{\prime}\left(w_{i_{0}}^{\dagger}\right)\right)}} \\
& \times \frac{1}{\left|\operatorname{det}\left(\left(1-w_{i_{0}}^{\dagger}\right) \mid \operatorname{Im}\left(1-w_{i_{0}}^{\dagger}\right)\right)\right|} \frac{1}{*\left({ }^{\prime} \mathscr{C}_{i_{0}}\left(w_{i_{0}}^{\dagger}\right)\right) \bullet *\left(\mathscr{C}_{i_{0}}^{\dagger^{\prime}}\right)} \\
& \times \int_{\sqrt{-1} a_{i_{0}}^{\prime}\left(w_{i_{0}}^{\dagger}\right)}\left\langle\mathbf{p}\left(\Gamma: \mathscr{C}_{i}: \mathbf{H}-\mathbf{H}_{\mathrm{O}}\right),\right. \\
& \operatorname{tr}_{\mathscr{C}_{i}}\left(\sum_{j_{0},{ }^{\prime} w_{j_{0} i_{0}}} D_{*}^{w_{j_{0}}^{\dagger}}\left[\mathbf{c}\left(P_{i_{0}}\left|A_{i_{0}}: P_{j_{0}}\right| A_{j_{0}}:{ }^{\prime} w_{j_{0} i_{0}}^{-1}: ?\right) \bullet \mathbf{e}\left(\mathscr{C}_{j_{0}}: \mathbf{H}_{\mathrm{O}}: ?\right)\right]{ }_{w_{j_{0} i_{0}}} \Lambda_{i_{0}}^{\prime}\right. \\
& \times \mathbf{c}\left(P_{j_{0}}\left|A_{j_{0}}: P_{i_{0}}\right| A_{i_{0}}:{ }^{\prime} w_{j_{0} i_{0}}: \Lambda_{i_{0}}^{\prime}\right) \\
& \left.\left.\times \mathbf{c}\left(P_{i_{0}}\left|A_{i_{0}}: P_{i_{0}}\right| A_{i_{0}}: w_{i_{0}}^{\dagger}: 0\right) \bullet \operatorname{Ind}_{\mathscr{C}_{i_{0}}}^{G}\left(\left(\mathscr{O}_{i_{0}}, \Lambda_{i_{0}}^{\prime}\right)\right)(\alpha)\right)\right\rangle\left|d \Lambda_{i_{0}}^{\prime}\right| .
\end{aligned}
$$

\section{CONTRIBUTION FROM THE CONTINUOUS SPECTRUM}

The purpose of this section is to consider the nature of the terms figuring in the contribution to the trace arising from the continuous spectrum. 
As it stands, modulo $\S 6$ infra, the Selberg trace formula asserts that for any $K$ central, $K$-finite element $\alpha$ of $C_{c}^{\infty}(G), \operatorname{tr}\left(L_{G / \Gamma}^{\text {dis }}(\alpha)\right)$ is equal to $\mathbf{K}(\mathbf{H}: \alpha: \Gamma)$ less $\mathbf{F n c}\left(\mathbf{H}: \mathbf{H}_{\mathrm{O}}: \alpha: \Gamma\right)$, explicated in Theorem 4.3. Denote by Con-Sp $(\mathbf{H}: \alpha: \Gamma)$ the result of setting $\mathbf{H}_{\mathrm{O}}=\mathbf{H}$ in $\mathbf{F n c}\left(\mathbf{H}: \mathbf{H}_{\mathrm{O}}: \alpha: \Gamma\right)$. Since

$$
\mathbf{p}\left(\Gamma: \mathscr{C}_{i}: \mathbf{0}\right)=0 \text {, }
$$

unless $\mathscr{C}_{i}=\{G\}$, only the undaggered terms from Theorem 4.3 remain. We can therefore regard $\operatorname{Con}-\operatorname{Sp}(\mathbf{H}: \alpha: \Gamma)$ as an infinite series

$$
\sum_{\mathscr{C}_{i_{0}}} \sum_{\mathscr{O}_{i_{0}}} \sum_{w_{i_{0}}} \operatorname{Con}-\mathbf{S p}\left(\mathbf{H}: \alpha: \Gamma: \mathscr{C}_{i_{0}}, \mathscr{O}_{i_{0}}, w_{i_{0}}\right)
$$

indexed by triples $\left(\mathscr{C}_{i_{0}}, \mathscr{O}_{i_{0}}, w_{i_{0}}\right)\left(w_{i_{0}}\right.$ simple $)$, there being a formula for

$$
\operatorname{Con}-\mathbf{S p}\left(\mathbf{H}: \alpha: \Gamma: \mathscr{C}_{i_{0}}, \mathscr{O}_{i_{0}}, w_{i_{0}}\right)
$$

that, apart from computable constants, is an integral over $\sqrt{-1} \operatorname{Ker}\left(1-w_{i_{0}}\right)$ of a totally explicit function. This function depends in general on $\mathbf{H}$. Of course, Con-Sp $(\mathbf{H}: \alpha: \Gamma)$ itself is a polynomial in $\mathbf{H}$.

Remark. Two special cases were dealt with in $\S 10$ of [11] by different methods, viz. when $w_{i_{0}}=1$ or $\operatorname{det}\left(1-w_{i_{0}}\right) \neq 0$. Those results agree with the ones obtained here.

The presence of $\mathbf{H}$ in $\operatorname{Con}-\operatorname{Sp}(\mathbf{H}: \alpha: \Gamma)$ is, perhaps, unexpected but not totally surprising. Indeed, such a parameter appears already in the invariant form of c-functions and Eisenstein series (cf. [12]), hence it is reasonable that such should survive into the trace formula too. However, in certain situations it is unnecessary.

Let us call $\Gamma$ regular at the cusps if there exists a choice of $\Gamma$-conjugacy class representatives $\left\{P_{i \mu}\right\}$ such that $\forall i_{0}, \mu_{0}$ :

$$
P \succeq P_{i_{0} \mu_{0}} \Rightarrow P=P_{i \mu} \quad(\exists i, \mu) .
$$

And let us call $\Gamma$ quasi-regular at the cusps if there exists a choice of $K$ and a choice of $\Gamma$-conjugacy class representatives $\left\{P_{i \mu}\right\}$ such that

$$
\exists \mathbf{0} \in \mathfrak{a} \text { s.t. } \forall i, \mu: \mathbf{0}\left(P_{i \mu}\right)=0 .
$$

It is clear that if $\Gamma$ is regular at the cusps, then it is quasi-regular at the cusps. All rank-1 $\Gamma$ are regular at the cusps. Every $\Gamma$ having just a single cusp is necessarily regular at that cusp. One can construct examples of $\Gamma$ in $\mathbf{S L}_{2}(\mathbf{R}) \times \mathbf{S L}_{2}(\mathbf{R})$ that are quasi-regular at the cusps but not regular at the cusps. Presumably, similar techniques would allow one to exhibit $\Gamma$ that are not quasiregular at the cusps but we have not checked this, although the usual choice $K=\mathrm{SO}_{2} \times \mathrm{SO}_{2}$ will not do.

When $\Gamma$ is quasi-regular at the cusps, the formula for

$$
\operatorname{Con}-\operatorname{Sp}\left(\mathbf{H}: \alpha: \Gamma: \mathscr{C}_{i_{0}}, \mathscr{O}_{i_{0}}, w_{i_{0}}\right)
$$


simplifies since one can take $\mathbf{H}_{\mathrm{O}}=\mathbf{0}$, thereby eliminating the exponential. In particular, this applies to the adele picture: Our methods can be transferred to that setting, the upshot being a sharpening of Arthur's results (cf. $[4, \S 8])$.

\section{ERROR ANALYSIS}

The purpose of this section is to prove that

$$
\sum_{\mathscr{E}_{i}, \mathscr{E}_{i_{0}}} \sum_{\mathscr{O}_{i_{0}}} \int_{\operatorname{Re}\left(\Lambda_{i_{0}}\right)=0}\left\langle\mathbf{p}\left(\Gamma: \mathscr{C}_{i}: \mathbf{H}-\mathbf{H}_{\mathrm{O}}\right), \mathbf{o}_{\mathscr{E}_{i}}\left(\mathscr{O}_{i_{0}}, \Lambda_{i_{0}}: \mathbf{H}\right)\right\rangle\left|d \Lambda_{i_{0}}\right|
$$

is $\mathbf{o}(\mathbf{H})$. To this end, we shall begin by showing that the problem, when considered along rays extending to infinity, can be recast so as to become one in real analysis. Once this is done, a fusion of the techniques from [9] (specifically, pp. 364-365) leads to its solution. The general case then follows readily.

Our starting point will be the function

$$
\mathbf{I}_{\alpha}\left(\mathscr{C}_{i}: \mathscr{O}_{i_{0}}, \Lambda_{i}+\Lambda_{i_{0}}^{\dagger}: \mathbf{H}\right)
$$

introduced in $\S 9$ of [11], to which we refer the reader for the definitions. In Lemma 9.2 of this reference it was established that

$$
\int_{\operatorname{Re}\left(\Lambda_{i}\right)=0} \sum_{\mathscr{\theta}_{i_{0}}} \int_{\operatorname{Re}\left(\Lambda_{i_{0}}^{\dagger}\right)=0}\left\|\mathbf{I}_{\alpha}\left(\mathscr{C}_{i}: \mathscr{O}_{i_{0}}, \Lambda_{i}+\Lambda_{i_{0}}^{\dagger}: \mathbf{H}\right)\right\|\left|d \Lambda_{i_{0}}^{\dagger} \| d \Lambda_{i}\right|<+\infty .
$$

We shall now sharpen this assertion. Write (cf. TES, p. 315)

$$
\mathscr{E}\left(\delta, \mathscr{O}_{i_{0}}\right)=\sum_{\mathscr{O}\left(i_{0}\right)} \sum_{\mathscr{E}} \bigoplus \mathscr{E}\left(\delta, \mathscr{O}_{i_{0}}\right) \cap \mathscr{E}\left(\delta, \mathscr{O}\left(i_{0}\right), \mathfrak{E}: P_{i_{0}} \mid A_{i_{0}}\right) .
$$

Choose an orthonormal basis for $\mathscr{E}\left(\delta, \mathscr{O}_{i_{0}}\right)$ compatible with the summands in this decomposition. Then attached to each such is an

$$
\mathbf{I}_{\alpha}\left(\mathscr{C}_{i}: \mathscr{O}_{i_{0}}: \mathscr{O}\left(i_{0}\right), \mathfrak{E}, \Lambda_{i}+\Lambda_{i_{0}}^{\dagger}: \mathbf{H}\right),
$$

the sum of which is

$$
\mathbf{I}_{\alpha}\left(\mathscr{C}_{i}: \mathscr{O}_{i_{0}}, \Lambda_{i}+\Lambda_{i_{0}}^{\dagger}: \mathbf{H}\right)
$$

And, since the convergence argument mentioned above only requires the use of convolution invariant subspaces,

$$
\begin{aligned}
\int_{\operatorname{Re}\left(\Lambda_{i}\right)=0} \sum_{\mathscr{O}_{i_{0}}} \int_{\operatorname{Re}\left(\Lambda_{i_{0}}^{\dagger}\right)=0} \sum_{\mathscr{O}\left(i_{0}\right)} \sum_{\mathscr{E}} \\
\quad \times\left\|\mathbf{I}_{\alpha}\left(\mathscr{C}_{i}: \mathscr{O}_{i_{0}}: \mathscr{O}\left(i_{0}\right), \mathfrak{E}, \Lambda_{i}+\Lambda_{i_{0}}^{\dagger}: \mathbf{H}\right)\right\|\left|d \Lambda_{i_{0}}^{\dagger} \| d \Lambda_{i}\right|<+\infty .
\end{aligned}
$$

Split, as is possible (cf. [11, Proposition 9.1]),

$$
\mathbf{I}_{\alpha}\left(\mathscr{C}_{i}: \mathscr{O}_{i_{0}}: \mathscr{O}\left(i_{0}\right), \mathfrak{E}, \Lambda_{i}+\Lambda_{i_{0}}^{\dagger}: \mathbf{H}\right)
$$


into $\mathbf{0}_{\mathscr{C}_{i}}\left(\mathscr{O}_{i_{0}}: \mathscr{O}\left(i_{0}\right), \mathfrak{E}, \Lambda_{i_{0}}: \mathbf{H}\right)$ plus $\amalg_{\phi}$, where $\phi$ is a certain Schwartz Detroit family. Thanks to Proposition 4.6 of [11], $\mathrm{W}_{\phi}$ is again Schwartz, hence is integrable. It therefore follows that

$$
\sum_{\mathscr{O}_{i_{0}}} \sum_{\mathscr{O}\left(i_{0}\right)} \sum_{\mathscr{E}} \int_{\operatorname{Re}\left(\Lambda_{i_{0}}\right)=0}\left\|\boldsymbol{o}_{\mathscr{C}_{i}}\left(\mathscr{O}_{i_{0}}: \mathscr{O}\left(i_{0}\right), \mathfrak{E}, \Lambda_{i_{0}}: \mathbf{H}\right)\right\|\left|d \Lambda_{i_{0}}\right|<+\infty .
$$

The main results from [8] allow one to say what

$$
\mathbf{0}_{\mathscr{C}_{i}}\left(\mathscr{O}_{i_{0}}: \mathscr{O}\left(i_{0}\right), \mathfrak{E}, \Lambda_{i_{0}}: \mathbf{H}\right)
$$

looks like. Indeed, Proposition 7.3 of that paper implies that

$$
\begin{aligned}
\mathbf{0}_{\mathscr{C}_{i}}\left(\mathscr{O}_{i_{0}}: \mathscr{O}\left(i_{0}\right), \mathfrak{E}, \Lambda_{i_{0}}: \mathbf{H}\right) \\
\quad=\sum_{j} q_{j}\left(\Lambda_{i_{0}}: \mathbf{H}\left(P_{j}\right)\right) \bullet \exp \left[\left\langle X_{j}+T_{j}\left(\Lambda_{i_{0}}\right), \mathbf{H}\left(P_{j}\right)\right\rangle\right],
\end{aligned}
$$

where $j$ runs over a finite index set, the corresponding $P_{j}$ representing (with overlap) the $\Gamma$-cuspidals associate to $P\left(i_{0}\right)$ below $P_{i_{0}}$. We can thus assume outright that the index set is fixed, independent of $\mathscr{O}_{i_{0}}, \mathscr{O}\left(i_{0}\right)$, and $\mathfrak{E}, 1 \leq j \leq$ $N$.

Turning first to the exponential, the $T_{j}$ are linear transformations

$$
T_{j}\left(\Lambda_{i_{0}}\right)=\left(w_{1}-w_{2}\right) \Lambda_{i_{0}}^{\dagger},
$$

with $w_{1}, w_{2} \in W\left(A_{j}, A\left(i_{0}\right)\right)\left(P\left(i_{0}\right) \preceq P_{i_{0}}\right)$. So, there are a fixed finite number of possibilities for them. On the other hand (cf. Theorem 6.5 and 8.6 of [8]), the $X_{j}$ are nonzero and lie in the closure of the negative cone of $\left(P_{j}, S_{j} ; A_{j}\right)$ (cf. TES, p. 71), there being a fixed finite number of possibilities for them too. Of course,

$$
X_{j}=X_{j}\left(\mathscr{O}_{i_{0}}: \mathscr{O}\left(i_{0}\right), \mathfrak{E}\right) .
$$

But $\left\|X_{j}\right\|^{-1}$ is a slowly increasing function of $\left\|\mathscr{O}_{i_{0}}\right\|$.

The $q_{j}\left(\Lambda_{i_{0}}: \mathbf{H}\left(P_{j}\right)\right)$ are, for each fixed $\Lambda_{i_{0}}$, a polynomial in $\mathbf{H}\left(P_{j}\right)$. If a $P_{j}$ or a $T_{j}$ does not arise, then $q_{j} \equiv 0$. We claim that in any event the degrees of the $q_{j}$ are uniformly bounded in the parameters. That this is the case is a consequence of $[9, \S 5]$. In fact, owing to Lemma 5.1 of [9], the degree of each $q_{j}$ is bounded by $\operatorname{rank}(\Gamma)+2$ times the maximum of the degrees of the constant term polynomials. To get a handle on the latter, one can use the argument of Proposition 5.2 in [9]. Strictly speaking, this argument was given there just for $L^{2}$-automorphic forms. However, it goes through with virtually no change for the constant term polynomials of automorphic forms that are linear combinations of $\mathfrak{Z}$-eigenvectors. In particular, it works for Eisenstein series (cuspidal or residual), from which the claim.

Keeping to the notation introduced in $\S 3$, we can and will assume that

$$
\mathbf{H}=\mathbf{H}_{00}+t \mathbf{H}_{0} \quad(t \geq 0),
$$


where $\mathbf{H}_{0} \in-\mathbf{C}_{0}(\cdot)$. There is no loss of generality in assuming further that if $X_{j} \neq X_{j^{\prime}}^{\prime}$ and $P_{j}=P_{j^{\prime}}$, then

$$
\left\langle X_{j}, \mathbf{H}_{0}\left(P_{j}\right)\right\rangle \neq\left\langle X_{j^{\prime}}^{\prime}, \mathbf{H}_{0}\left(P_{j^{\prime}}\right)\right\rangle .
$$

Accordingly,

$$
\begin{aligned}
& \mathbf{o}_{\mathscr{C}_{i}}\left(\mathscr{O}_{i_{0}}:\right.\left.\mathscr{O}\left(i_{0}\right), \mathfrak{E}, \Lambda_{i_{0}}: \mathbf{H}\right) \\
&=\sum_{j} q_{j}\left(\Lambda_{i_{0}}: \mathbf{H}_{00}\left(P_{j}\right)+t \mathbf{H}_{0}\left(P_{j}\right)\right) \\
& \quad \bullet \exp \left[\left\langle X_{j}+T_{j}\left(\Lambda_{i_{0}}\right), \mathbf{H}_{00}\left(P_{j}\right)\right\rangle\right] \\
& \quad \exp \left[t\left\langle X_{j}+T_{j}\left(\Lambda_{i_{0}}\right), \mathbf{H}_{0}\left(P_{j}\right)\right\rangle\right]
\end{aligned}
$$

Put

$$
\begin{aligned}
q_{j}\left(\Lambda_{i_{0}}: t\right)= & q_{j}\left(\Lambda_{i_{0}}: \mathbf{H}_{00}\left(P_{j}\right)+t \mathbf{H}_{0}\left(P_{j}\right)\right) \\
& \bullet \exp \left[\left\langle X_{j}+T_{j}\left(\Lambda_{i_{0}}\right), \mathbf{H}_{00}\left(P_{j}\right)\right\rangle\right],
\end{aligned}
$$

a polynomial in $t$ of uniformly bounded degree. If we set $r_{j}=\left\langle X_{j}, \mathbf{H}_{0}\left(P_{j}\right)\right\rangle$ and $v_{j}=T_{j}^{*}\left(\mathbf{H}_{0}\left(P_{j}\right)\right)$, then

$$
\begin{aligned}
\mathbf{0}_{\mathscr{C}_{i}}\left(\mathscr{O}_{i_{0}}: \mathscr{O}\left(i_{0}\right), \mathfrak{E}, \Lambda_{i_{0}}: \mathbf{H}\right) \\
\quad=\sum_{j} q_{j}\left(\Lambda_{i_{0}}: t\right) \bullet \exp \left[t\left(r_{j}+\left\langle\Lambda_{i_{0}}, v_{j}\right\rangle\right)\right] .
\end{aligned}
$$

Note that the $r_{j}$ depend on the parameters but the $v_{j}$ do not.

Indexing the data subject to increasing $\mathscr{O}_{i_{0}}$ and then relabeling leads to the following situation: A function $\phi(\Lambda, t)$ on $\mathbf{R}^{n} \times \mathbf{R}_{\geq 0}$ given as an infinite series

$$
\phi(\Lambda, t)=\sum_{k=1}^{\infty} \phi_{k}(\Lambda, t),
$$

where each $\phi_{k}(\Lambda, t)$ has the form

$$
\sum_{j=1}^{N} \sum_{l=0}^{L} a_{j k l}(\Lambda) t^{l} \bullet \exp \left[t\left(r_{j k}+\sqrt{-1}\left\langle\Lambda, v_{j}\right\rangle\right)\right] ;
$$

here $N$ and $L$ are independent of $k$ and

$$
\left\{\begin{array}{l}
r_{j k}<0, \\
-r_{j k} \geq C k^{-N}
\end{array}\right.
$$

for $C$ some absolute constant. The $v_{j}$ are vectors in $\mathbf{R}^{n}$. We know that

$$
\int_{\mathbf{R}^{n}} \sum_{k=1}^{\infty}\left|\phi_{k}(\Lambda, t)\right| d \Lambda<+\infty \quad \forall t \geq 0 .
$$

From all this, we want to conclude that

$$
\int_{\mathbf{R}^{n}} \phi(\Lambda, t) d \Lambda \rightarrow 0 \text { as } t \rightarrow+\infty .
$$


Actually, as can be seen by example, what is formally before us is not quite enough to force the desired deduction. Fortunately, though, a little bit more is available in that we have not made use of the other terms figuring in the definition of $\mathbf{I}_{\alpha}$, terms about which a greal deal is known.

To isolate the essential point, a definition is in order, namely: Call a sequence $\left\{f_{k}(\Lambda)\right\}$ of measurable functions on $\mathbf{R}^{n}$ almost rapidly decreasing if $\forall N>0$,

$$
\sum_{k=1}^{\infty} k^{N} \int_{\mathbf{R}^{n}}\left|f_{k}(\Lambda)\right| \cdot(1+\|\Lambda\|)^{N} d \Lambda<+\infty
$$

It is clear that

$$
\text { rapidly decreasing } \Rightarrow \text { almost rapidly decreasing }
$$

but not conversely.

Proposition 6.1. The sequence $\left\{\left\|\phi_{k}(\Lambda, ?) \mid[0,1]\right\|_{1}\right\}$ is almost rapidly decreasing. Proof. Retranslated, the problem is to prove that

$$
\left\|\mathbf{0}_{\mathscr{C}_{i}}\left(\mathscr{O}_{i_{0}}: \mathscr{O}\left(i_{0}\right), \mathfrak{E}, \Lambda_{i_{0}}: \mathbf{H}_{00}+? \mathbf{H}_{0}\right) \mid[0,1]\right\|_{1}
$$

is almost rapidly decreasing in $\left(\mathscr{O}_{i_{0}}, \Lambda_{i_{0}}\right)$. Consider the expression for

$$
\mathbf{I}_{\alpha}\left(\mathscr{C}_{i}: \mathscr{O}_{i_{0}}: \mathscr{O}\left(i_{0}\right), \mathfrak{E}, \Lambda_{i}+\Lambda_{i_{0}}^{\dagger}: \mathbf{H}_{00}+? \mathbf{H}_{0}\right)
$$

as the sum of $\mathbf{0}_{\mathscr{C}_{i}}\left(\mathscr{O}_{i_{0}}: \mathscr{O}\left(i_{0}\right), \mathfrak{E}, \Lambda_{i_{0}}: \mathbf{H}_{00}+? \mathbf{H}_{0}\right)$ and $\amalg_{\phi}$. Since there are no difficulties with $\amalg_{\phi}$, we shall concentrate on $\mathbf{I}_{\alpha}$. Employing the usual artifice, it can be supposed that $\alpha=\beta * \beta^{*}$. From positive definiteness, $\mathbf{I}_{\alpha}$ is then monotone increasing in $t$, hence

$$
\begin{aligned}
\left\|\mathbf{I}_{\alpha}\left(\mathscr{C}_{i}: \cdots: \mathbf{H}_{00}+? \mathbf{H}_{0}\right)\right\|[0,1] \|_{1} \\
\quad \leq\left\|\mathbf{I}_{\alpha}\left(\mathscr{C}_{i}: \cdots: \mathbf{H}_{00}+\mathbf{H}_{0}\right)\right\| .
\end{aligned}
$$

To see that the latter majorant is almost rapidly decreasing in $\left(\mathscr{O}_{i_{0}}, \Lambda_{i_{0}}\right)$, one has only to bring in the Casimir and argue as in Lemma 9.3 of [11].

Recall that our objective is to prove that

$$
\lim _{t \rightarrow+\infty} \int_{\mathbf{R}^{n}} \phi(\Lambda, t) d \Lambda=0 .
$$

To accomplish this, it will be necessary to combine the foregoing proposition with a lemma on exponential polynomials.

Lemma 6.2. Suppose that

$$
E(t)=\sum_{j=1}^{N} Q_{j}(t) \bullet \exp \left(-t s_{j}\right)
$$

is an exponential polynomial, where

(1) $\forall j, \operatorname{deg}\left(Q_{j}\right) \leq L$ and

(2) $\forall j, s_{j} \in \mathbf{C \& R e}\left(s_{j}\right) \in[0,1],\left|\operatorname{Im}\left(s_{j}\right)\right| \leq A$. 
Then there exists $M_{0}>0$, depending on $N$ and $L$ but not on $A$, such that $\forall t \geq 0$,

$$
|E(t)| \leq M_{0}(1+A)^{N(L+1)}(1+t)^{N(L+1)-1}\|E \mid[0,1]\|_{1} .
$$

Granted the lemma, we can finish the discussion. Thus put

$$
\begin{gathered}
Q_{j k}(\Lambda, t)=\sum_{l=0}^{L} a_{j k l}(\Lambda) t^{l}, \\
s_{j k}=-C k^{-N}-r_{j k}-\sqrt{-1}\left\langle\Lambda, v_{j}\right\rangle, \\
A=\max _{j}\left\|v_{j}\right\| \bullet\|\Lambda\| .
\end{gathered}
$$

Then

$$
\begin{aligned}
\phi_{k}(\Lambda, t) & =\sum_{j=1}^{N} \sum_{l=0}^{L} a_{j k l}(\Lambda) t^{l} \bullet \exp \left[t\left(r_{j k}+\sqrt{-1}\left\langle\Lambda, v_{j}\right\rangle\right)\right] \\
& =\left(\sum_{j=1}^{N} Q_{j k}(\Lambda, t) \bullet \exp \left(-t s_{j k}\right)\right) \bullet \exp \left(-C k^{-N} t\right) \\
& =E_{k}(\Lambda, t) \bullet \exp \left(-C k^{-N} t\right) .
\end{aligned}
$$

By adjusting $\mathbf{H}_{0}$ (cf. TES, p. 157), matters can be arranged to ensure that $-1 \leq r_{j k}<0$. Consequently, $1>-C k^{-N}-r_{j k} \geq 0$. The lemma is therefore applicable and, setting $N_{0}=N(L+1)$, we find that $\forall t \geq 0$,

$$
\begin{aligned}
\left|\phi_{k}(\Lambda, t)\right| \leq & \exp \left(-C k^{-N} t\right) \\
& \times M_{0}^{\prime}(1+\|\Lambda\|)^{N_{0}}(1+t)^{N_{0}}\left\|E_{k}(\Lambda, ?) \mid[0,1]\right\|_{1} \\
& \leq M_{0}^{\prime \prime}(1+t)^{N_{0}} \exp \left(-C k^{-N} t\right) \\
& \bullet(1+\|\Lambda\|)^{N_{0}}\left\|\phi_{k}(\Lambda, ?) \mid[0,1]\right\|_{1}
\end{aligned}
$$

and so

$B_{k}$ being

$$
\int_{\mathbf{R}^{n}}\left|\phi_{k}(\Lambda, t)\right| d \Lambda \leq(1+t)^{N_{0}} \exp \left(-C k^{-N} t\right) \bullet B_{k},
$$

$$
M_{0}^{\prime \prime} \bullet \int_{\mathbf{R}^{n}}(1+\|\Lambda\|)^{N_{0}}\left\|\phi_{k}(\Lambda, ?) \mid[0,1]\right\|_{1} d \Lambda,
$$

a rapidly decreasing function of $k$ (cf. Proposition 6.1). Bearing in mind that

$$
\phi(\Lambda, t)=\sum_{k=1}^{\infty} \phi_{k}(\Lambda, t),
$$

to finish it obviously suffices to show that

$$
\sum_{k=1}^{\infty} B_{k} \bullet \exp \left(-C k^{-N} t\right)
$$


is rapidly decreasing in $t \geq 0$. For this purpose, let $\eta$ be any positive integer. Then

$$
\begin{aligned}
& t^{\eta} \sum_{k=1}^{\infty} B_{k} \bullet \exp \left(-C k^{-N} t\right) \\
& \quad=\frac{\eta^{\eta}}{C^{\eta}} \cdot \sum_{k=1}^{\infty} k^{\eta N} B_{k} \cdot\left[\frac{C k^{-N} t}{\eta} \exp \left(-\frac{C k^{-N} t}{\eta}\right)\right]^{\eta} \\
& \quad \leq \frac{\eta^{\eta}}{(e C)^{\eta}} \cdot \sum_{k=1}^{\infty} k^{\eta N} B_{k}<+\infty,
\end{aligned}
$$

which does the trick.

Let us now pass to the proof of the lemma. The key to the whole business is the special case $A=1$, viz.:

Sublemma. Under the above assumptions, suppose in addition that $\forall j, \operatorname{Im}\left(s_{j}\right) \in$ $[-1,1]$. Then there exists $M_{0}>0$, depending on $N$ and $L$, such that $\forall t \geq 0$,

$$
|E(t)| \leq M_{0}(1+t)^{N(L+1)-1}\|E \mid[0,1]\|_{1} .
$$

Proof. We shall use the Laplace transform techniques of [9], establishing, in fact, that the estimate obtains for all functions whose Laplace transforms have the form

$$
\frac{P(s)}{\prod_{j=1}^{N_{0}}\left(s+s_{j}\right)} \quad\left(s_{j} \in[0,1]+\sqrt{-1}[-1,1]\right),
$$

where $P \in$ Poly $_{N_{0}}$, the polynomials of degree $<N_{0}=N(L+1)$. Given an $N_{0}$-tuple

$$
\mathbf{s} \in([0,1]+\sqrt{-1}[-1,1])^{N_{0}},
$$

define transformations

$$
\left\{\begin{array}{l}
T_{\mathbf{s}}^{\prime}: \text { Poly }_{N_{0}} \rightarrow S I_{N_{0}-1}\left(\mathbf{R}_{\geq 0}\right), \\
T_{\mathbf{s}}^{\prime \prime}: \text { Poly }_{N_{0}} \rightarrow L^{1}([0,1])
\end{array}\right.
$$

by the simultaneous prescription

$$
P \rightarrow \operatorname{Lap}^{-1}\left(\frac{P(s)}{\prod_{s_{j} \in \mathbf{s}}\left(s+s_{j}\right)}\right) .
$$

We explicitly observe that our definitions are meaningful in that both invert Laplace transforms but the results are considered in different function spaces. Since

$$
\left\{T_{\mathbf{s}}^{\prime}: \mathbf{s} \in([0,1]+\sqrt{-1}[-1,1])^{N_{0}}\right\}
$$

is uniformly bounded, $\exists M^{\prime}>0$ such that $M^{\prime} \geq\left\|T_{\mathbf{s}}^{\prime}\right\|(\forall \mathbf{s})$. Moreover, in view of what can be found on p. 365 of [9], $\exists M^{\prime \prime}>0$ such that

$$
\left\|T_{\mathbf{s}}^{\prime \prime}(P)\right\|_{1} \geq M^{\prime \prime}\|P\| \quad(\forall \mathbf{s}, P)
$$

or still

$$
\|E \mid[0,1]\|_{1} \geq M^{\prime \prime}\|P\| \quad \text { if } E=T_{\mathbf{s}}^{\prime}(P) .
$$


The sup over $t \geq 0$ of $(1+t)^{-N_{0}+1}|E(t)|$ is the norm in $S I_{N_{0}-1}\left(\mathbf{R}_{\geq 0}\right)$ of $E$, hence

$$
(1+t)^{-N_{0}+1}|E(t)| \leq\left\|T_{\mathbf{s}}^{\prime}(P)\right\| \leq M^{\prime}\|P\| \leq M^{\prime} / M^{\prime \prime} \bullet\|E \mid[0,1]\|_{1},
$$

so $M_{0}=M^{\prime} / M^{\prime \prime}$ will work.

To treat the case of arbitrary $A$, we shall simply scale the data, taking $A>1$. Thus let

$$
\widetilde{E}(t)=E(t / A)=\sum_{j=1}^{N} Q_{j}(t / A) \bullet \exp \left(-t s_{j} / A\right) .
$$

Then (cf. supra)

$$
|\widetilde{E}(t)| \leq M_{0}(1+t)^{N(L+1)-1}\|\widetilde{E} \mid[0,1]\|_{1}
$$

implies

$$
|E(t)|=|\widetilde{E}(A t)| \leq M_{0}(1+A t)^{N(L+1)-1}\|\widetilde{E} \mid[0,1]\|_{1} .
$$

Finally,

$$
\begin{aligned}
\|\widetilde{E} \mid[0,1]\|_{1} & =\int_{0}^{1}|E(t / A)| d t \\
& =A \int_{0}^{1 / A}|E(t)| d t \leq(1+A)\|E \mid[0,1]\|_{1} .
\end{aligned}
$$

Consequently,

$$
\sum_{\mathscr{C}_{i}, \mathscr{E}_{i_{0}}} \sum_{\mathscr{O}_{i_{0}}} \int_{\operatorname{Re}\left(\Lambda_{i_{0}}\right)=0}\left\langle\mathbf{p}\left(\Gamma: \mathscr{C}_{i}: \mathbf{H}-\mathbf{H}_{\mathrm{O}}\right), \mathbf{o}_{\mathscr{C}_{i}}\left(\mathscr{O}_{i_{0}}, \Lambda_{i_{0}}: \mathbf{H}\right)\right\rangle\left|d \Lambda_{i_{0}}\right|
$$

is $\mathbf{o}(\mathbf{H})$.

\section{REFERENCES}

1. J. Arthur, The characters of discrete series as orbital integrals, Invent. Math. 32 (1976), 205-261.

2.

3. $\ldots$, On a family of distributions obtained from Eisenstein series. I, Amer. Math. 104 (1982), 1243-1288.

4. __, On a family of distributions obtained from Eisenstein series. II, Amer. J. Math. 104 (1982), 1289-1336.

5. M. S. Osborne and G. Warner, The Selberg trace formula. I, Crelle's J. 324 (1981), 1-113.

6. __ The Selberg trace formula. II, Pacific J. Math. 106 (1983), 307-496.

7. _ The Selberg trace formula. III, Mem. Amer. Math. Soc. No. 283 (1983), 1-209.

8. _ The Selberg trace formula. IV, Lecture Notes in Math., vol. 1024, Springer, 1983, pp. 112-263.

9. __ The Selberg trace formula. V, Trans. Amer. Math. Soc. 286 (1984), 351-376.

10. _ The Selberg trace formula. VI, Amer. J. Math. 107 (1985), 1369-1437.

11. _ The Selberg trace formula. VII, Pacific J. Math. 140 (1989), 263-352.

12. P. Ringseth, Invariant Eisenstein systems, Ph. D. Thesis, University of Washington, 1986. 